\title{
Characterisation of strain localisation processes during fatigue crack initiation and early crack propagation by SEM-DIC in an advanced disc alloy
}

\author{
R. Jiang*, F. Pierron, S. Octaviani, P.A.S. Reed
}

Materials Research Group, Faculty of Engineering and the Environment, University of Southampton, Highfield, Southampton, SO17 1BJ, UK

*Corresponding author. Tel: +44 (0)23 80594438; Fax: +44 (0)23 80593016;

Email: Rong.Jiang@soton.ac.uk

\begin{abstract}
:
Fatigue failure processes in metallic materials are closely related to the evolution of strain localisation under cyclic loading. Characterisation of this strain localisation is important in understanding the mechanisms of fatigue crack initiation and propagation, and provides critical validation data to develop appropriate crystal plasticity models for prediction of these processes. In this study, strain localisation during fatigue crack initiation and early crack propagation in an advanced Ni-based superalloy for turbine disc application has been characterised at the grain level with a sub-micron resolution by digital image correlation on SEM images using secondary $\gamma^{\prime}$ themselves as the speckle pattern. The obtained full-field strains have been analysed in global coordinates associated with the applied loading direction and in terms of the local coordinates associated with individual slip bands. Deformation arising from in-plane and out-of-plane dislocation slip can be identified by a combination of shear strain $\varepsilon_{\mathbf{x y}}$ and transverse strain $\varepsilon_{\mathbf{y y}}$ in the local slip band coordinates in combination with EBSD analysis. Cracks preferentially initiate from slip/strain bands adjacent and parallel to twin boundaries and then propagate along the slip/strain bands, leading to the onset of significant transverse strain $\boldsymbol{\varepsilon}_{\mathbf{y y}}$ in the local band coordinates as a consequence of crack opening. Crack propagation is closely related to strain accumulation at the crack tip which is determined by the grain orientation and grain size. Transverse strain $\boldsymbol{\varepsilon}_{\mathbf{y y}}$ in local slip band coordinates together with the inclination angle between dislocation slip direction on an activated $\{111\}$ plane and the slip trace of this $\{111\}$ plane at the specimen surface is proposed to be a cracking indicator/fracture criterion.
\end{abstract}

Keywords: Ni-based superalloys; Strain localisation; Digital image correlation; Fatigue cracking; Transverse strain 


\section{Introduction}

Powder metallurgy (PM) Ni-based superalloys have been widely used for high pressure disk rotor applications in aeroengines due to their excellent combined properties, i.e. high strength at elevated temperatures, good resistance to fatigue, creep, oxidation and corrosion [1-3]. Among all the properties of PM Ni-base superalloys for disk applications, fatigue resistance is one of the most important, often limiting the overall service life. It is generally accepted that fatigue crack initiation and short crack growth processes are important to optimise as they contribute to the majority of fatigue life of a turbine disc during service. This is due to the high overall component stresses which results in a relatively small extent of fatigue crack propagation prior to fast fracture and thereby limits the fatigue life dependency to the fatigue crack initiation and short crack growth regime [4-8]. In the course of service, disc alloys are subjected to complex loading conditions across the disc section at a temperature range of $400{ }^{\circ} \mathrm{C}-750{ }^{\circ} \mathrm{C}$, which usually results in intragranular fatigue crack initiation at the disc bore where the material is subjected to lower temperatures but higher stresses [8-11] and intergranular fatigue crack initiation at the disc rim due to stress/strain assisted grain boundary oxidation at higher temperatures [12-15]. Extensive studies have shown that fatigue crack initiation is usually found at slip bands/crystallographical facets $[8$, $11,16]$, pores $[4,17]$ and especially twin boundaries (TBs) $[8,16,18]$ at the relatively lower temperatures in the disc bore region. This is closely related to the intrinsic deformation behaviour of the disc alloys with insignificant/limited environmental damage. Once a crack initiates, it is observed to propagate along a crystallography facet or grain boundary (GB) in the early stages of crack growth, with a strong interaction with microstructural features. This is also affected by the loading conditions and the development of the plastic zone/accumulated strain at the crack tip $[4,5,10,16,19]$.

Significant efforts have been made by many researchers to understand the mechanisms of these fatigue cracking processes under a wide range of different loading conditions via experimental studies $[4,6,8,10,11,16]$ and to predict the crack initiation and propagation in disc alloy/components using crystal plasticity finite element (CPFE) models [20-25]. To understand these fatigue failure mechanisms and to develop and validate these CPFE models, one missing requirement has been the quantitative assessment of the actual localised strains at the grain level and their dependency on grain orientation and the neighbouring grains/microstructures under the relevant in-service temperatures and loading conditions [21, $22,25,26]$. However, quantitative measurement of full-field strain distribution at a grain 
level is still quite challenging. For instance, accumulated inelastic strain at the crack tip has been used as a fracture criterion in a CPFE model to predict crack propagation in disc alloy RR1000 based on Karabela's study [21], but the experimental validation of the accumulated inelastic strain at a grain level at the crack tip is still not in place, which limits the further development of these CPFE models.

Although X-ray and neutron diffraction have been well established to measure the elastic strains in materials, this averages out the information in the whole activated volume [27-30]. Recently, electron backscatter diffraction (EBSD) has been developed to characterise the residual elastic strain with a high spatial resolution based on the shift of the zone axis of Kikuchi pattern in the materials/specimen due to the improvement of angular resolution during sampling of the Kikuchi pattern [23, 31-36]. Meanwhile, the geometrically necessary dislocation (GND) distribution can also be calculated based on the lattice curvature [32, 35, 36]. This technique appears to be valuable to capture the small levels of residual elastic strains, especially in the single crystal material, in which the reference Kikuchi pattern can be chosen from far field strain-free regions and is applicable to whole sampling area. In terms of polycrystalline alloys however, this incurs a problem of choosing the reference Kikuchi pattern due to the misorientation between different grains. The chosen reference Kikuchi pattern within the sampling area in the polycrystalline alloys is essentially in an unknown strain state (although normally the reference pattern is chosen from the least deformed region) [35-37]. As a consequence of the "reference pattern problem", the measured residual elastic strain only represents the relative deformation within the same grain in polycrystalline materials. In addition, this technique is unable to capture a larger plastic strain field. Although the global strain is usually small in fatigue testing, the localised strain at certain microstructural features, e.g. GBs and TBs, can be much higher than the global strain $[9,38$, 39]. Therefore, other complementary techniques are needed to characterise the localised strain evolution during the fatigue cracking process.

Digital image correlation (DIC) is a well-established and robust tool used to measure fullfield in-plane strain by correlating a random speckle pattern in images taken before and after deformation [26, 38-46]. A displacement field is initially obtained via this image correlation process, and then a strain field can be derived through numerical differentiation. Generally, the images used for DIC are captured by an optical camera, which means that it is not possible to achieve sub-micron resolution at a grain level in polycrystalline disc alloys with grain sizes between 10-50 $\mu \mathrm{m}[39,41,44,45,47]$. By using scanning electron microscopy 
(SEM) to take the images for DIC, the pixel resolution can be significantly improved, thus allowing the characterisation of strain distribution at the grain scale, although the process of SEM imaging itself may cause additional error/noise (i.e. time varying distortion due to image drift at high magnification and spatial distortion at low magnification) due to beam rastering when taking an image [26, 38, 43, 48-51]. Even though in SEM the pixel resolution of the images used for DIC is compatible with the grain size, the obtained strain resolution is still inherently limited by speckle size [47]. As a general rule, a DIC subset needs to contain at least three speckles, therefore, very fine speckles need to be produced for SEM-DIC. In recent publications, Fabio Di Gioacchino et al. [26, 43] applied nanometer size gold particles on a stainless steel surface via a re-modelling process, and achieved a strain spatial resolution at the sub-micron scale in an ex-situ tensile test. They showed that the strain localisation exists in the form of bands (similar to slip bands) within grains under monotonic tensile loads. This concentrated strain in bands was shown to be consistent with the slip traces of the $\{111\}<110>$ slip systems expected based on a combined analysis with EBSD. Stinville et al. $[9,38,52]$ also achieved a similar strain resolution at sub-micron scale in a Ni-based superalloy using secondary $\gamma^{\prime}$ as a natural speckle under tension and compression loading, and found that strain localised at the slip bands adjacent and parallel to TBs. However, all these studies using SEM-DIC to investigate strain localisation were mainly conducted under monotonic loads, few studies have been carried out under cyclic loading which may involve the slip reversal associated with back and forth dislocation movement [53]. This is clearly different from the deformation seen under purely monotonic loading. In addition, the interpretation of the axial, transverse and shear strain levels in these studies under monotonic loading was performed only in the global coordinates associated with the loading direction, which cannot directly link the obtained bands of concentrated strain to the crystallography of the investigated materials and dislocation slip behaviour, although the slip traces of $\{111\}<110>$ slip systems and Schmid factor (SF) analysis were carried out based on the EBSD investigation of grain orientation.

In the present study, strain localisation at the grain level with a sub-micron resolution during fatigue crack initiation and early crack propagation regimes in an advanced disc alloy, i.e. Low Solvus, High Refractory (LSHR) alloy, has been investigated by SEM-DIC using secondary $\gamma^{\prime}$ as the speckles at room temperature. The strain development/increment at each interruption throughout an interrupted fatigue test has been captured. Annealing twins are a pertinent microstructural feature in PM Ni-based superalloys. Our previous studies and 
studies by other groups have shown that cracks mainly initiate at TB or in slip bands adjacent and parallel to $\mathrm{TB}$ in large twin-containing grains with high $\mathrm{SF}[8,16,38]$. Strain accumulation at/nearby TBs under cyclic load is therefore of particular interest to the present study. The obtained strain field has been analysed in both the global coordinates associated with the loading direction and the local coordinates associated with individual slip band to provide insights of the damage arising and evolving from dislocation slip under cyclic loading. Applicability of the transverse strain in the local slip band coordinates along with the inclination angle between dislocation slip direction on $\{111\}$ plane and slip trace (i.e. intersection line of $\{111\}$ slip plane on the specimen surface) as a cracking indicator has been discussed.

\section{Materials and experimental procedures}

\subsection{Materials}

The LSHR alloy used in this study was provided by NASA. Composition (in wt.\%) of the LSHR alloy is $12.5 \mathrm{Cr}, 20.7 \mathrm{Co}, 2.7 \mathrm{Mo}, 3.5 \mathrm{Ti}, 3.5 \mathrm{Al}, 0.03 \mathrm{C}, 0.03 \mathrm{~B}, 4.3 \mathrm{~W}, 0.05 \mathrm{Zr}, 1.6 \mathrm{Ta}$, $1.5 \mathrm{Nb}$, Ni bal. Specimens used for short crack fatigue tests were extracted from a turbine disc which was fabricated by canning atomized LSHR alloy powder followed by hot isostatically pressing, extruding and isothermally forging. The extracted specimens were supersolvus heat treated at $1171{ }^{\circ} \mathrm{C}$ for 1 hour followed by a cooling of $72{ }^{\circ} \mathrm{C} / \mathrm{min}$ to dissolve primary $\gamma^{\prime}$ and to yield coarse grained microstructures. Subsequently the specimens were aged at $855{ }^{\circ} \mathrm{C}$ for 4 hours and then $755^{\circ} \mathrm{C}$ for 8 hours to modify the precipitation of secondary $\gamma^{\prime}$. The obtained microstructural features of the LSHR alloy are summarized in Table 1 as reported in our previous study [3].

Table 1 Grain size and $\gamma^{\prime}$ size in the LSHR alloy

\begin{tabular}{ccccc}
\hline Materials & Grain size range $(\mu \mathrm{m})$ & Average grain size $(\mu \mathrm{m})$ & Primary $\gamma^{\prime}(\mu \mathrm{m})$ & Secondary $\gamma^{\prime}(\mathrm{nm})$ \\
\hline LSHR & $10-140$ & $36.1 \pm 18.1$ & N/A & $153 \pm 29$ \\
\hline
\end{tabular}

\subsection{Fatigue tests}

Fatigue tests were conducted on plain bend bar $(4 \mathrm{~mm} \times 4 \mathrm{~mm} \times 55 \mathrm{~mm})$ specimens under three point bend loading on an Instron 8501 hydraulic testing machine at room temperature with a $20 \mathrm{~Hz}$ sine waveform and a load ratio of 0.1 . The distance between the top two rollers is $40 \mathrm{~mm}$ with a roller located at the bottom at the mid-point. The applied load was chosen to produce a maximum total strain of $\sim 0.007$ (or maximum plastic strain of $\sim 0.0015$ ) on the top central surface based on a finite element elasto-plastic simulation. The set-up of the three 
point bending test is schematically shown in Fig. 1 (a). The specimens were ground with 120 grit, 800 grit, 1200 grit and 4000 grit SiC abrasive papers, and then polished with $0.05 \mu \mathrm{m}$ colloidal silica. Before the fatigue test, EBSD was employed to map the grain orientations on the top central surface using Channel $5 \mathrm{HKL}$ software. A grain boundary tolerance angle of $2^{\circ}$ and a step size of $2 \mu \mathrm{m}$ were employed for EBSD mapping. In order to remove the residual surface deformation layer after mechanical polishing to obtain high indexation rate during EBSD mapping, the specimens were immersed in Kalling's reagent $(80 \mathrm{ml} \mathrm{HCl}+40 \mathrm{ml}$ $\mathrm{CH}_{3} \mathrm{OH}+40 \mathrm{~g} \mathrm{CuCl}_{2}$ ) for $1 \mathrm{~s}$. The specimen longitudinal direction was aligned with the stage $\mathrm{X}$ axis when conducting the EBSD. The corresponding highest SF in each grain was calculated based on the EBSD measurement and was used as a criterion to choose the area of interest (AOI) for the following investigation of strain localisation by SEM-DIC under cyclic loading. The obtained SF map overlaid with GBs and TBs is shown in Fig. 1 (b). EBSD measurements were also used to calculate and plot the slip traces of $\{111\}$ planes in the investigated grains. Seven areas which contain large grains with high SF and a twin boundary were chosen for the study of strain localisation in the crack initiation regime. The chosen AOIs for study of strain localisation are highlighted in Fig. 1 (b).

(a)

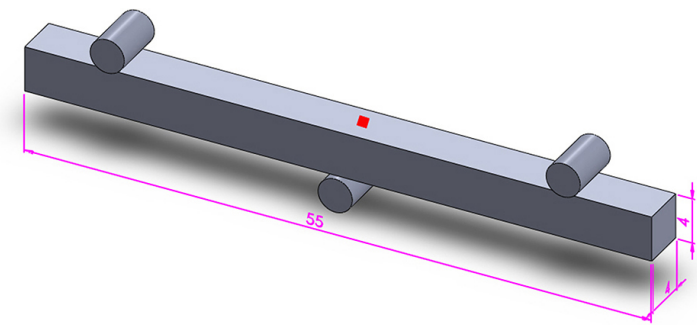

(b)

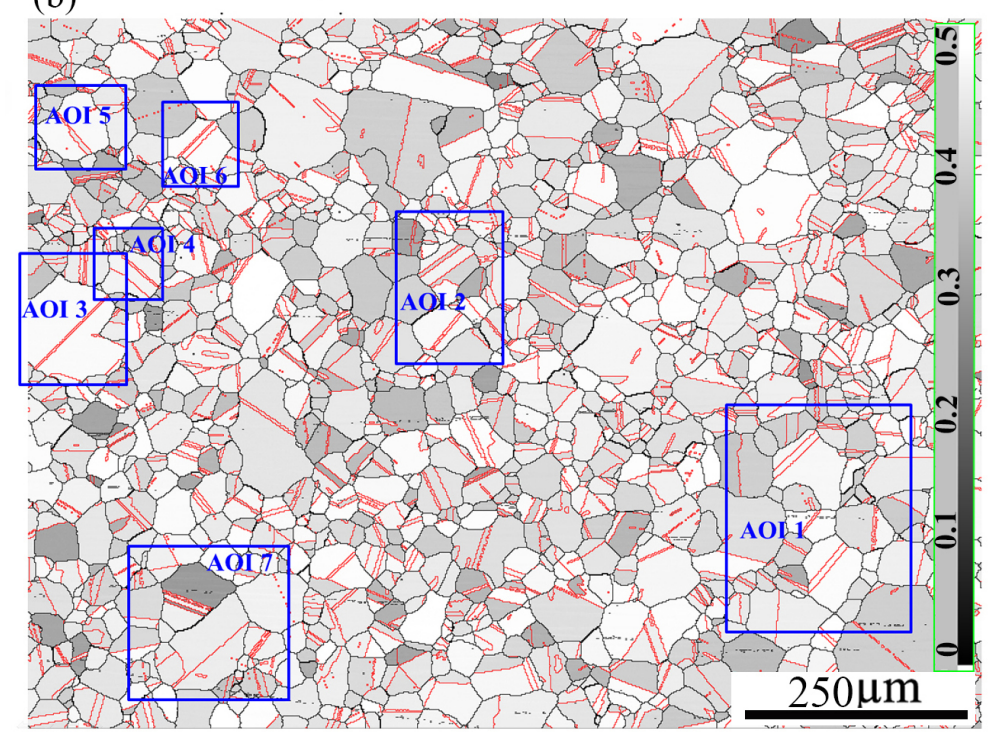

(c)
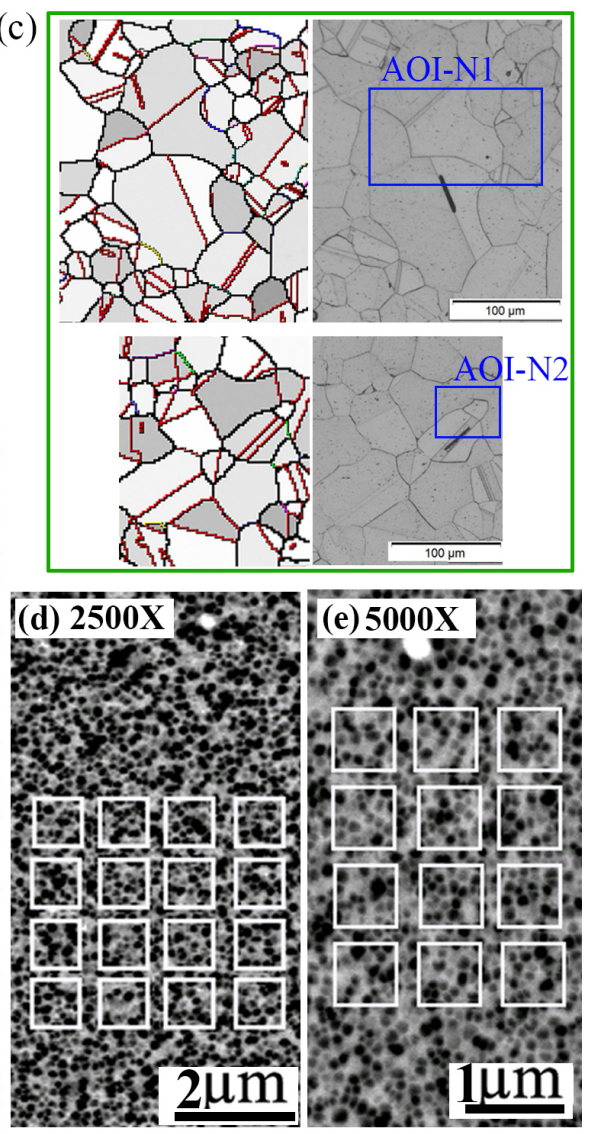
Fig. 1 (a) Schematic diagram of three point bending test. The red rectangle points out the location of AOIs for EBSD and SEM-DIC analysis. (b) Schmid factor map overlaid with grain boundaries (black lines) and twin boundaries (red lines) in the specimen without FIBed notch. (c) Schmid factor maps and optical microscopy images of the AOIs containing FIBed notches. (d) and (e) Morphology of secondary $\gamma^{\prime}$ (i.e. speckles for DIC) in LSHR alloy under BEI mode at magnifications of $2500 \times$ and $5000 \times$ respectively. The subsets used for DIC analysis are indicated by the white squares. For interpretation of the references to colour in this figure legend, the reader is referred to the web version of this article.

To evaluate the strain localisation processes at the crack tip during crack propagation, notches were introduced into the top central surface in another specimen by focussed ion beam (FIB) and thus acted as artificial cracks to overcome the uncertainty of capturing the natural crack initiation sites, so as to have specific areas for SEM-DIC investigation of strain accumulation at the crack tip during crack propagation. Two FIBed notches with a dimension of $30 \mu \mathrm{m} \times 1 \mu \mathrm{m} \times 10 \mu \mathrm{m}$ were made along the twin boundary, one was in large grain with high SF, and the other was in small grain with relatively low SF, which to provide insight into the influence of grain size and orientation on strain localisation and crack propagation. The prepared notches and the associated AOIs are shown in Fig. 1 (c).

After identifying the AOIs, the specimens were etched in Kalling's reagent for $30 \mathrm{~s}$ to reveal secondary $\gamma^{\prime}$ to provide a speckle pattern with good contrast for SEM-DIC analysis. Reference images were taken at the AOIs using a JEOL field gun emission SEM JSM6500F under the backscatter electron imaging (BEI) mode before the fatigue tests. The SEM imaging parameters used are shown in Table 2 following guidelines proposed by the literature $[43,48,49,51]$. It should be mentioned that a relatively lower magnification (i.e. $2500 \times$ ) was used in the specimen without FIBed notches to sample a larger area to allow the capture of sufficient crack initiation events, whereas a relatively higher magnification (i.e. $5000 \times$ ) was employed in the specimen with FIBed notches to better detect the crack propagation processes. The morphology of secondary $\gamma^{\prime}$ (i.e. speckles for DIC) at these two magnifications is shown in Figs. 1 (d) and (e) respectively. Once the reference images were taken, fatigue tests were conducted and interrupted after certain loading cycles to capture the SEM images in the ex-situ (unloaded) deformed AOIs. The load cycling history of each set of specimen observations is summarised in Table 3. 
Table 2 Imaging parameters in JOEL SEM JSM6500F

\begin{tabular}{cccccc}
\hline $\begin{array}{c}\text { Imaging } \\
\text { mode }\end{array}$ & $\begin{array}{c}\text { Acceleration } \\
\text { voltage }\end{array}$ & $\begin{array}{c}\text { Probe current } \\
\text { spot size }\end{array}$ & $\begin{array}{c}\text { Imaging } \\
\text { time }\end{array}$ & $\begin{array}{c}\text { Working } \\
\text { distance }\end{array}$ & Image size \\
\hline BEI & $15 \mathrm{kV}$ & 13 & $80 \mathrm{~s}$ & $8.7 \mathrm{~mm}$ & $1280 \times 1024$ \\
\hline
\end{tabular}

Table 3 Test/interruption history of the specimens

\begin{tabular}{ll}
\hline Specimen & Test/interruption history \\
\hline $\begin{array}{l}\text { LSHR without } \\
\text { notch }\end{array}$ & $\begin{array}{l}2000 \text { cycles } \rightarrow 7000 \text { cycles } \rightarrow 12000 \text { cycles } \rightarrow 16000 \text { cycles } \rightarrow 20000 \text { cycles } \rightarrow 21000 \\
\text { cycles } \rightarrow 23000 \text { cycles } \rightarrow 25000 \text { cycles } \rightarrow 28000 \text { cycles } \rightarrow 31000 \text { cycles- }\end{array}$ \\
\hline $\begin{array}{l}\text { LSHR } \\
\text { notch }\end{array}$ & with \\
\hline
\end{tabular}

\subsection{DIC analysis}

DIC analysis was carried out using MatchID commercial software. To better understand the obtained strain fields, noise assessment of the background strain induced by electron beam drift distortion and errors caused by electron beam re-positioning during SEM imaging was first assessed by pairs of consecutive stationary images. Noise assessment was performed at both magnifications of $2500 \times$ and $5000 \times$. Due to the ex-situ nature of the tests, the specimens had to be placed back into the SEM after ex-situ load cycling for each observation. Hence the strain noise arising from the successive re-positioning and observations of the specimens in SEM was also assessed by taking pairs of stationary images from the same area but in different SEM observation sessions (without any loading cycle increment).

The SEM images obtained from each AOI were stitched in the Fiji open source software, and then the stitched images were input into MatchID for DIC analysis. Subsets of $21 \times 21$ pixels $(0.798 \mu \mathrm{m} \times 0.798 \mu \mathrm{m}$ at $2500 \times)$ and $33 \times 33$ pixels $(0.627 \mu \mathrm{m} \times 0.627 \mu \mathrm{m}$ at $5000 \times)$ were used for the specimens with and without FIBed notches respectively. The different subset sizes arise from the fact that the material features are taken as speckles and therefore, since a subset needs to contain at least one speckle, the subset size needs to be adjusted with the magnification. Figs. 1 (d) and (e) show typical subset windows for the two magnifications. A step size of 7 pixels was used in both cases. This is a compromise between larger step sizes which are computationally efficient and smaller ones which would capture the local strain peaks more accurately. The zero-normalized sum of squared differences (ZNSSD) criterion, which accounts for offset and scaling in intensity variations of the investigated images, was adopted to perform the correlation in order to exclude the effects of contrast difference in the SEM images taken at different observation times. Deformed images were interpolated using a bicubic spline interpolation algorithm to obtain subpixel accuracies in the displacement 
calculation. Quadratic shape functions were used to expand the displacement field in the subset. This is a better choice than linear shape functions, as demonstrated in [54] and leads to enhanced spatial resolution. Once the displacement field was calculated, the Logarithmic Euler-Almansi strain fields were derived by performing a local bilinear polynomial fit in MatchID using a window of $3 \times 3$ data points (or a 'strain window' of 3). The smallest possible strain window was selected here to enhance spatial resolution at the cost of strain resolution. To document the effects of the employed DIC parameters on the obtained strain fields, Fig. 2 shows the $\varepsilon_{\mathrm{xx}}$ strain maps in a small region within AOI 3 after 2000 loading cycles. The reference map is (a) with a subset size of 21 , a step of 7 and a quadratic shape function. Changing the subset size to 15 (b) does not change the strain map significantly, and this is about the smallest subset that is feasible with the current configuration. However, changing the step size to 1 has a significant effect, increasing background noise but also providing larger localized peak strains. The Virtual Strain Gauge size, or VSG, represents the number of horizontal or vertical image pixels used to get a strain value, calculated as: $\mathrm{VSG}=(\mathrm{SW}-1) * \mathrm{ST}+\mathrm{SS}$ where $\mathrm{SW}$ is the strain window as defined above, ST is the step size and SS is the subset size [55]. So the smaller the VSG, the larger the noise and the better the spatial resolution. Interestingly, the change of VSG between (a) and (b) is the same as that between (b) and (c) so clearly, the VSG is not a $100 \%$ accurate representation of the level of filtering of the data. From the use of simulated deformed speckle images encoding a sinusoidal displacement, it was found that the step size had a major effect on where the maximal strain was sampled [55]. This is the effect that can be seen between (a) and (c). Finally, a parameter that is often overlooked in DIC studies is the shape function. This is the function that is used to expand the deformation of a subset. Even though it has been demonstrated long ago that quadratic shape functions were largely superior to linear ones [54], most commercial DIC codes still use linear shape functions. Looking at the difference between maps (a) and (d), the major effect of the shape function choice is clearly illustrated. Based on this analysis, it was chosen to keep a subset of 21 pixels, a step of 7 and a strain window of 3 . Peak strains would have been better captured with a step of 1 but these calculations are rather computationally intensive and since the current article will only make use of the data in a qualitative way, it was felt that this was appropriate. However, if such data were used for more quantitative assessments, then clearly a step of 1 would be necessary. Finally, all images show vertical stripes. These are spurious and specific to ex-situ SEM-DIC artefacts and will be documented further in Section 3.1. 
(a) Subset: 21 pixels; Step size: 7 pixels; (b) Subset: 15 pixels; Step size: 7 pixels; Bicublic Spline; Quadratic; VSG: 35 Bicublic Spline; Quadratic; VSG: 29
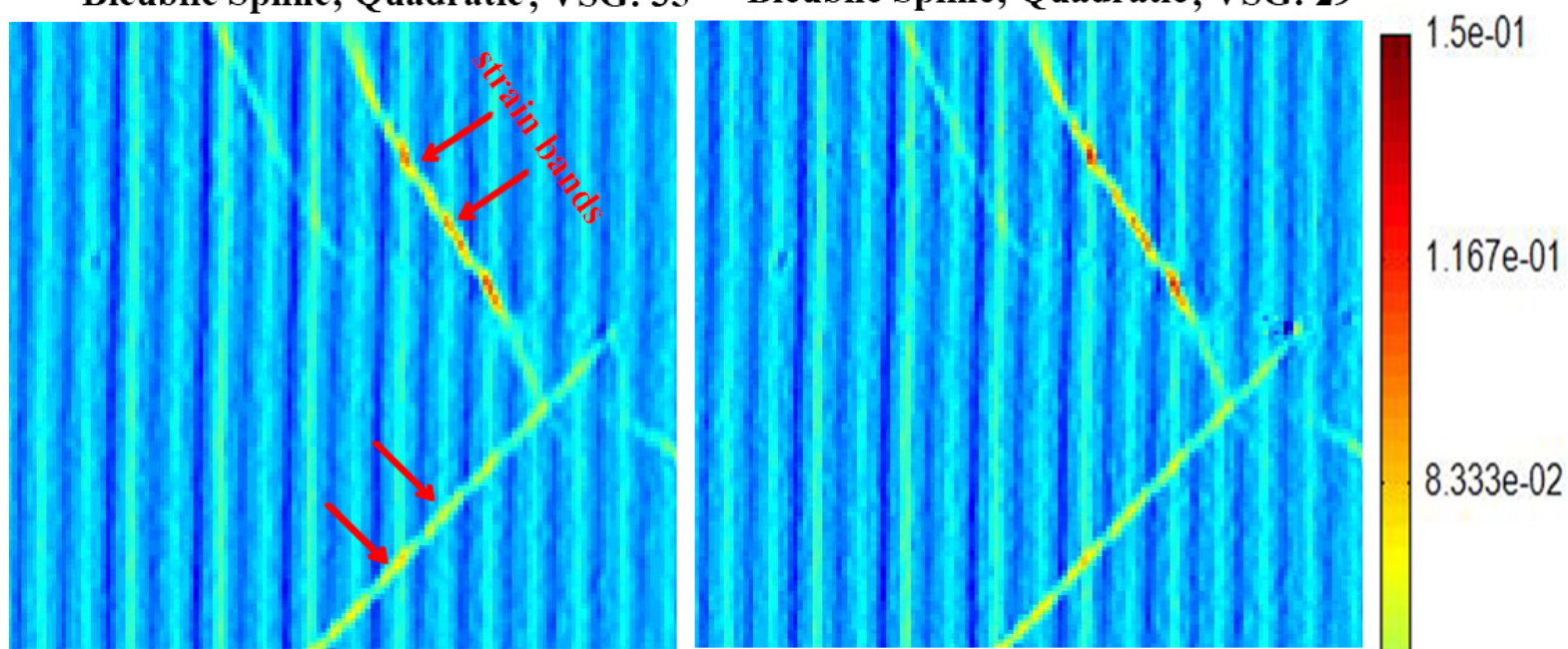

(c) Subset: 21 pixels; Step size: 1 pixels; Bicublic Spline; Quadratic; VSG: 23

(d) Subset: 21 pixels; Step size: 7 pixels;

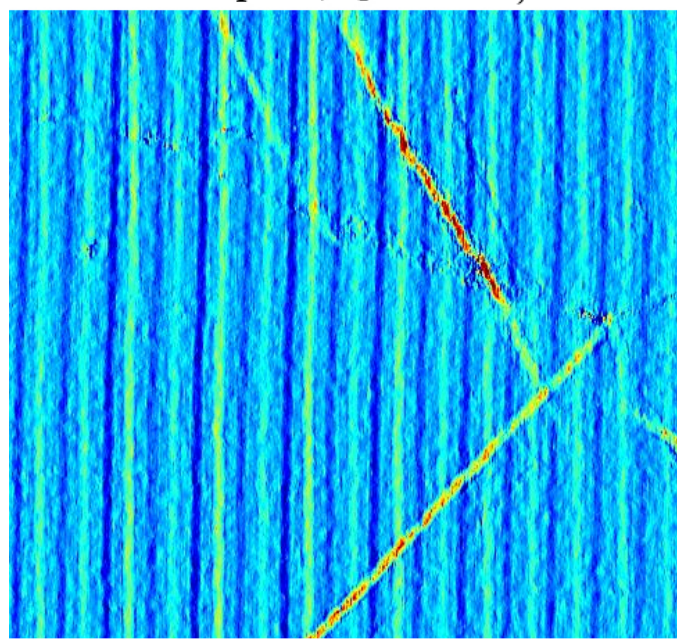

Bicublic Spline; Affine; VSG: 35

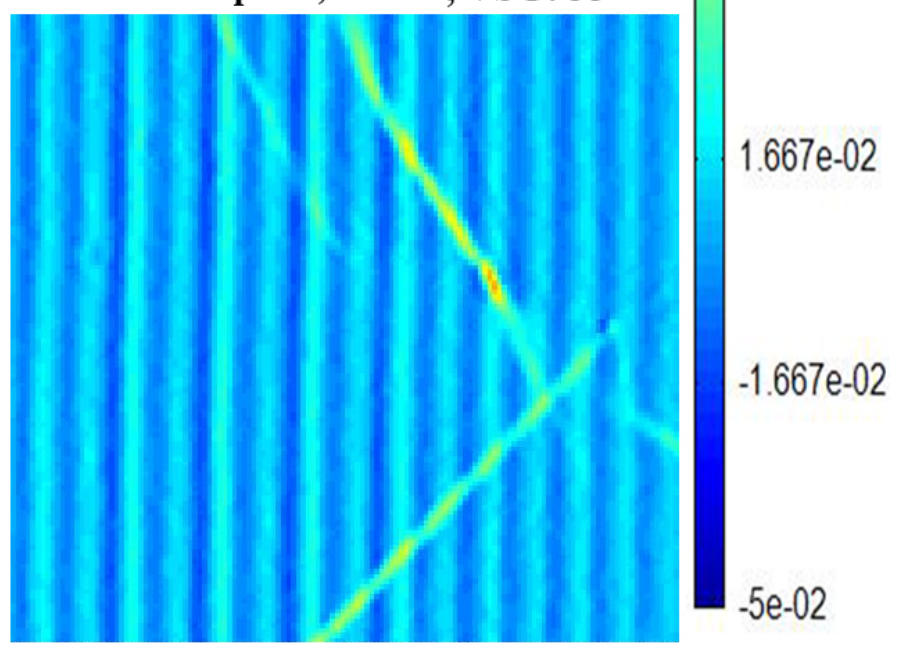

Fig. 2 Comparison of the effects of employed DIC parameters on obtained localised distribution of strain $\varepsilon_{\mathrm{xx}}$ in a small region within AOI 3 after 2000 loading cycles.

Since the deformation localizes in bands in these materials, the strains in the global specimen axes does not allow discrimination between shear bands and cracks. To do so, the strain fields will be rotated in the local coordinate system associated with each considered band. This is a novelty compared to a recent similar study [38] where only global coordinates were considered. Moreover, to represent the shear strains in a field of view containing bands of different orientations, the maximum shear strain will be considered. This is the radius of Mohr's circle and is independent of the coordinate system. Therefore, readings of maximum shear strains can be quantitatively compared between bands of different orientations. The maximum shear strain is given by the following equation: 
$\varepsilon_{x y}^{\max }=\sqrt{\left(\frac{\varepsilon_{x x}-\varepsilon_{y y}}{2}\right)^{2}+\varepsilon_{x y}^{2}}$

\section{Results}

\subsection{Noise assessment of SEM-DIC}

The noise assessment indicates that the background strain noise is on a level of $\sim \pm 1 \%$ for the image pair taken during the same observation session in the SEM at either $2500 \times$ or $5000 \times$, but it should be noted that re-positioning the specimens into the SEM may cause additional errors/noise as shown in Fig. 3. The left column in Fig. 3 displays the background noise obtained from the stationary image pair without re-positioning the specimen in the SEM. A random strain noise distribution can be seen in all the strain maps $\left(\varepsilon_{\mathrm{xx}}, \varepsilon_{\mathrm{yy}}, \varepsilon_{\mathrm{xy}}\right.$ and $\left.\varepsilon_{x y}^{\max }\right)$. The strain noise obtained from the image pair before and after re-positioning the specimen in the SEM (as shown in the right column in Fig. 3), is larger with a clear systematic pattern, i.e. vertical strain bands in the $\varepsilon_{\mathrm{xx}}$ map, horizontal strain bands in the $\varepsilon_{\mathrm{yy}}$ map and a grid pattern in the $\varepsilon_{x y}^{\max }$ map, and the strain noise level can be up to $\sim 3 \%$. It seems that re-positioning the specimen in the SEM has no apparent influence on $\varepsilon_{\mathrm{xy}}$. These vertical and horizontal strain bands are usually easy to recognize and hence do not significantly interfere with the identification of real strain localization events. A similar systematic noise pattern has also been seen at $5000 \times$. Although the noise seems to be high compared the global strain achieved by the applied load, a high enough signal/noise ratio can still be achieved as shown in the following localised strain results. This is therefore still an effective and robust tool to investigate the strain localisation processes. However, because of the high localization of the strain in bands, the values have to be considered as a lower bound of the actual ones. Indeed, DIC acts as a low-pass spatial filter, by analogy, it is like developing a finite element model with a fixed element size (the subset size), hence not being able to check for convergence. This is partially documented in the discussion attached to Fig. 2. Only increased magnification can eventually provide DIC convergence and actual strain values. This is a goal for future studies but in this case, artificial speckling will be necessary. 


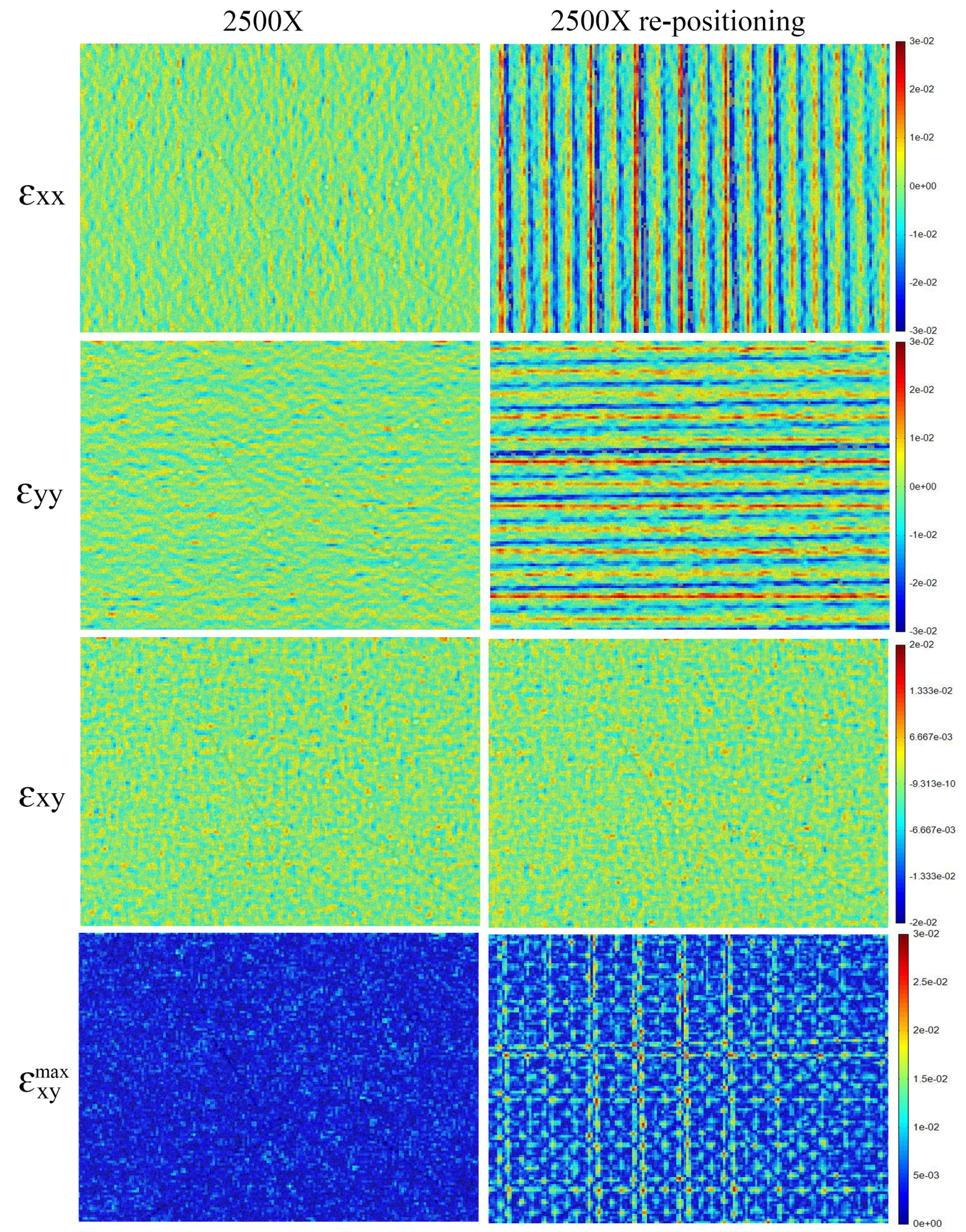

Fig. 3 Strain noise distribution from the stationary image pair without re-positioning the specimen in SEM (left column), and strain noise distribution from the image pair before and after re-positioning the specimen in SEM (right column). For interpretation of the references to colour in this figure legend, the reader is referred to the web version of this article.

\subsection{Strain localisation in the crack initiation regime}


Strain localisation during the fatigue crack initiation regime in all the 7 selected AOIs (as shown in Fig. 1 (b)) followed a similar pattern: strain localisation preferentially occurs in a large grain with high SF and adjacent and parallel to twin boundaries. In those grains with low SF, strain localisation is rarely seen, even though they also contain annealing twins. However, it should be noted that the obtained strain is the residual strain due to the ex-situ nature of the fatigue tests and subsequent observations. Fig. 4 (a) presents the stitched BEI images of AOI 3 before the fatigue test. TBs are indicated by the yellow arrows, which can also be seen in Fig. 1 (b). Slip traces of $\{111\}$ planes in grains 1 and 2 are plotted out based on EBSD measurement and calculation. The tensile stress direction is horizontal (the $\mathrm{X}$ axis of the global coordinates). As shown in Fig. 4 (b), strain accumulation adjacent and parallel to $\mathrm{TB}$ is indicated by the band of concentrated strain observed after first loading interruption, here denoted as strain band AB. Hereafter, this kind of strain accumulation showing features of a band of concentrated strain is termed a strain band (analogous to a slip band) for ease of description. Although the background strain noise can be as high as 3\% (as shown in Fig. 3) due to the ex-situ nature of the SEM-DIC conducted in this study, a good signal/noise ratio can be obtained as shown in the $\varepsilon_{x y}^{\max }$ map as the strains in the bands are much higher than 3\%. With further loading cycles, new strain bands (e.g. strain band CD) parallel to the TB appear with increasing strain (Fig. 4 (c)). These strain bands parallel to the TB are consistent with one of the calculated $\{111\}$ slip traces and are associated with the slip system with the highest SF in the twin-containing grain, and terminate at grain boundaries. Apart from the prominent strain bands parallel to the $\mathrm{TB}$, we can see another strain band (i.e. strain band $\mathrm{EF}$ ) located in grain 2 which penetrates through the grain boundary as shown in Figs. 4 (b) and (c). Strain distribution and evolution in strain bands $\mathrm{AB}$ and $\mathrm{CD}$ with successive loading cycles is shown in Figs. 4 (d) and (e) respectively, both of which show more severe strain localisation in the middle of the band at the centre of the grain. For strain band AB which is adjacent and parallel to the TB, it seems the strain saturates after the first test interruption (i.e. 2000 cycles) and no further increase in this residual strain value is seen in the following loading cycles. For strain band CD formed after 20000 cycles and parallel to TB, the strain increases continuously with the loading cycles until 28000 cycles, and then the residual strain shows a tendency of saturation at the end of the fatigue test (i.e. 31000 cycles). It appears that the strain is slightly higher in strain band $\mathrm{CD}$ than in strain band $\mathrm{AB}$.

To allow better understanding of the nature of these strain bands, the strain are re-plotted in their own local co-ordinates. Fig. 4 (f) displays the strain bands parallel to the TB in their 
local co-ordinates. The $\mathrm{X}$-axis is now parallel to the TB, and the defined coordinates are shown in Fig. 4 (f). It is found that these strain bands parallel to the TB show predominantly a shear nature indicated by the significant shear strain $\varepsilon_{\mathrm{xy}}$ and low $\varepsilon_{\mathrm{xx}}$ and $\varepsilon_{\mathrm{yy}}$. By more detailed observation of the strain band AB in the SEM, we can see sheared $\gamma^{\prime}$ precipitates in the strain band as shown in Fig. 4 (g) indicated by the arrows; this is a consequence of dislocation shear processes cutting the coherent $\gamma^{\prime}$. It can also be discerned that the sheared $\gamma^{\prime}$ precipitates are quite close to the TB. Strain distribution in strain band EF in its local coordinates is presented in Fig. 4 (h). It is seen that strain band EF has a dominant $\varepsilon_{y y}$ strain component along with a much smaller $\varepsilon_{x y}$ value, which is quite different from the strain distribution seen in the bands parallel to the TB. But similar to the strain bands parallel to the $\mathrm{TB}$, no apparent strain component of $\varepsilon_{\mathrm{xx}}$ can be found in strain band EF in terms of its local coordinates. The morphology of strain band EF is shown in Fig. 4 (i). Unlike the strain bands parallel to TB, no sheared $\gamma^{\prime}$ is observed in plane. It appears that instead material extrusion can be discerned indicated by the contrast difference between the band and $\gamma^{\prime}$ in the SEM image obtained using the secondary electron signal. The same contrast difference is also seen in the BEI image. 
(a)
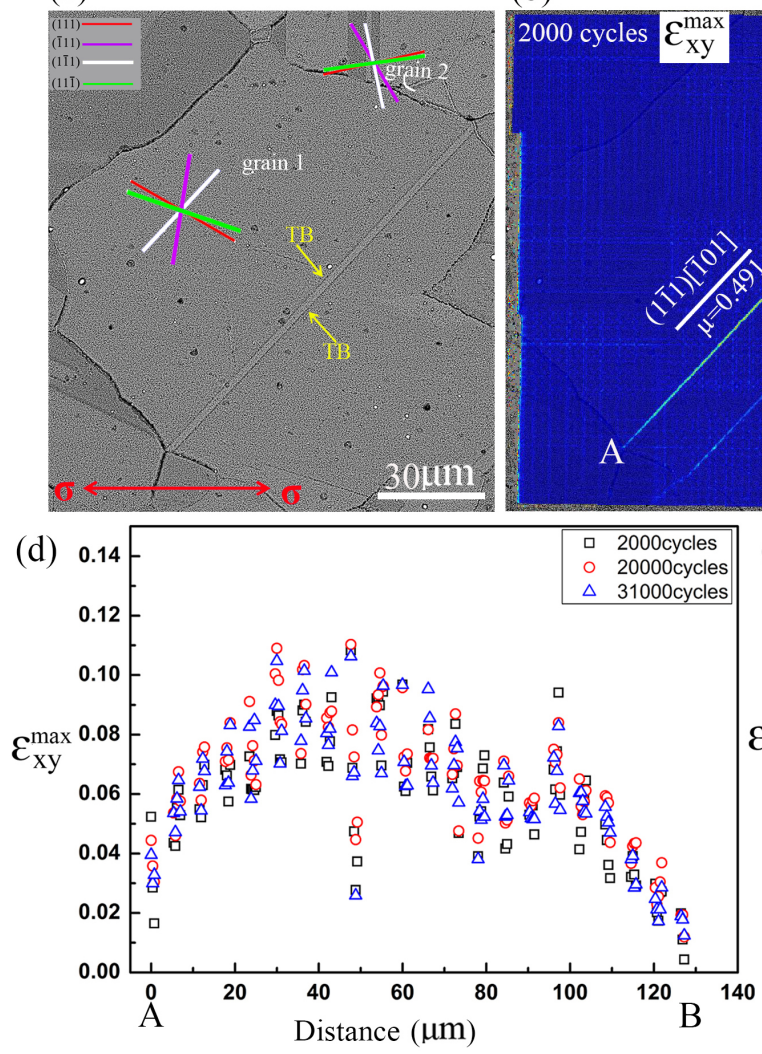

(b)
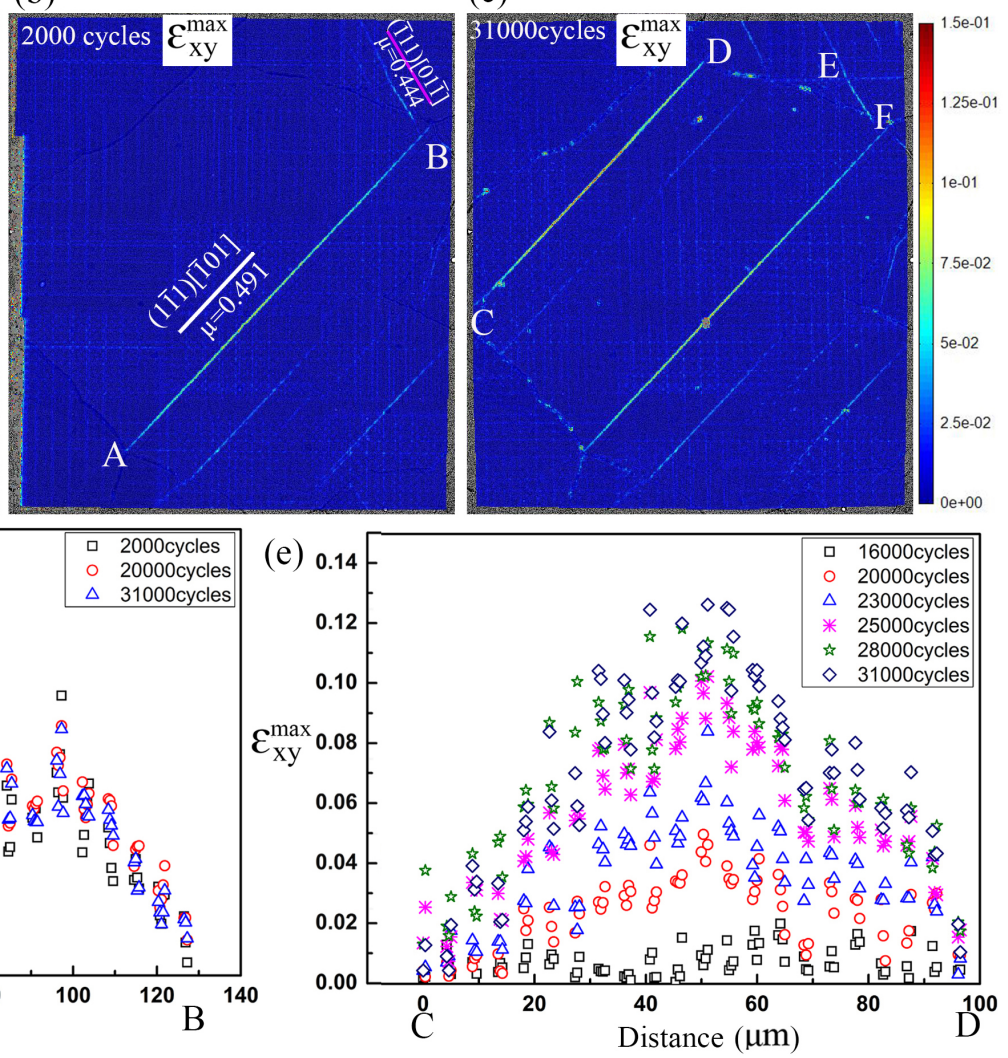

(c)

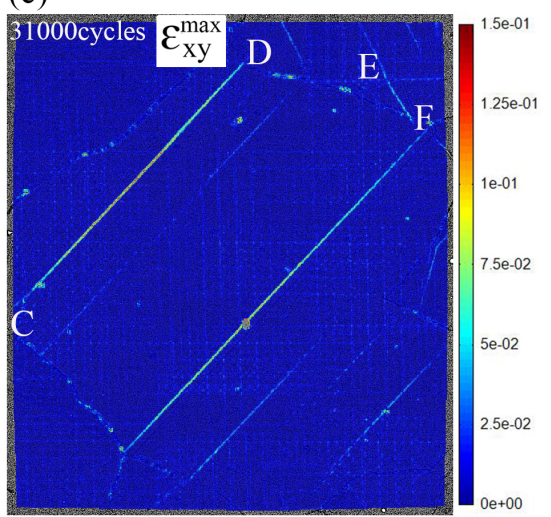

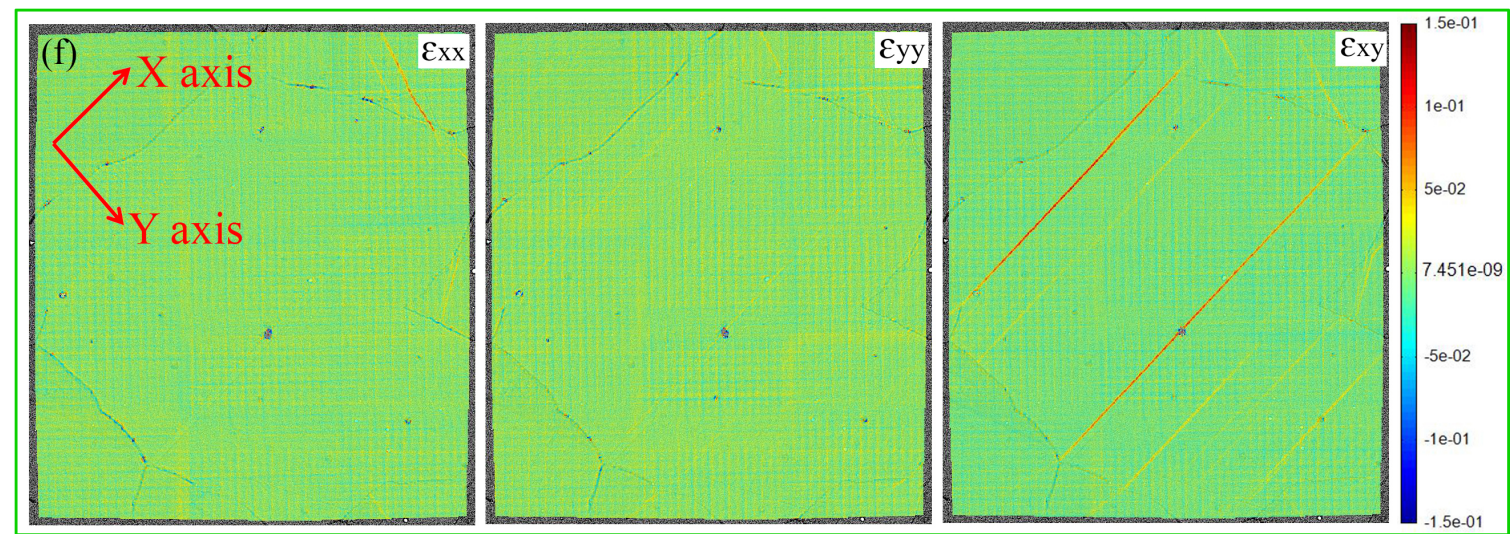

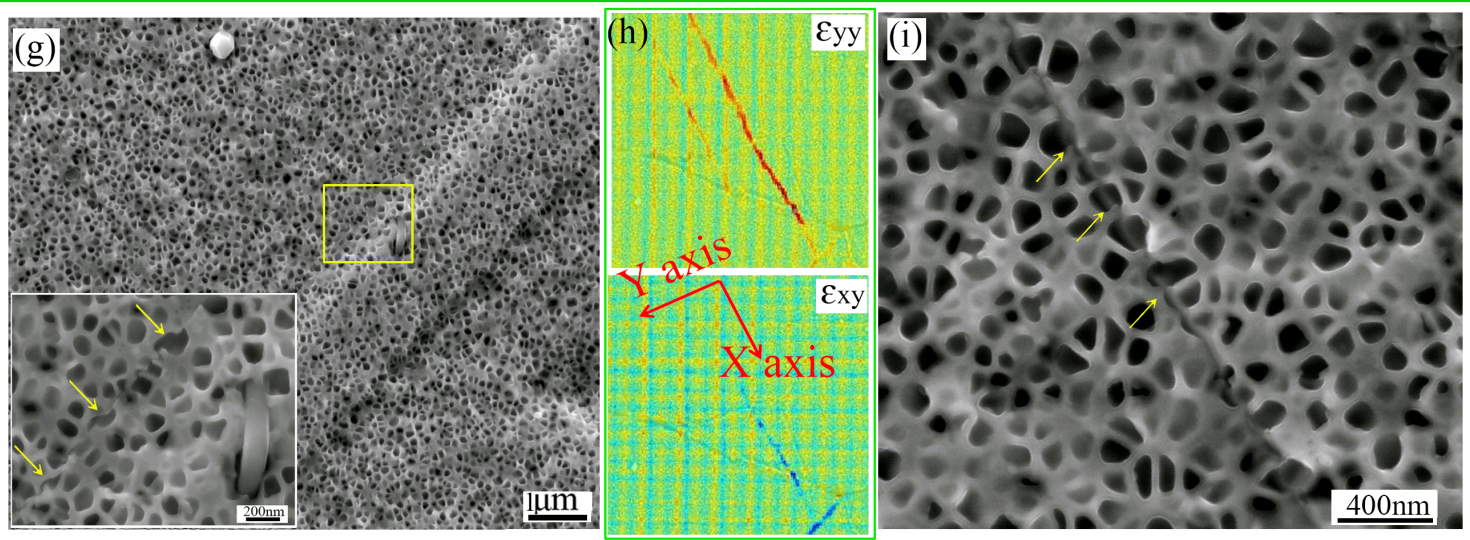

Fig. 4 (a) Stitched BEI images of AOI 3 before fatigue test. Slip traces of $\{111\}$ planes in grains 1 and 2 (which contain strain bands) are plotted out based on EBSD measurement and 
calculation. (b) and (c) Maximum shear strain $\varepsilon_{x y}^{\max }$ map at 2000 and 31000 loading cycles respectively. (d) and (e) maximum shear strain $\varepsilon_{x y}^{\max }$ distribution in strain bands $A B$ and $C D$ shown in (b) and (c) respectively. (f) Re-plotting of the strain bands (e.g. strain bands AB and $\mathrm{CD})$ parallel to the TB in their own local coordinates. The $\mathrm{X}$ axis is along the strain band. $(\mathrm{g})$ Microstructural features associated with strain band AB. (h) Re-plotting of strain band EF in its own local coordinates. (i) Microstructural features associated with strain band EF. For interpretation of the references to colour in this figure legend, the reader is referred to the web version of this article.

Fig. 5 shows the maximum shear strain $\varepsilon_{x y}^{\max }$ evolution with loading cycles in the AOI 7. The stitched SEM images before and at the end of the test are shown in Figs. 5 (a) and (i) respectively. The obtained GBs and TBs by EBSD measurement are overlaid on the SEM image in Fig. 5 (a), and the calculated slip traces of the $\{111\}$ planes are shown in Fig. 5 (i). Two cracks can be clearly seen at the end of the test, one (i.e. crack 1) is pretty straight and adjacent and parallel to the TB in grain 3, and the other one (i.e. crack 2) is macroscopically straight but microsopically deflected and has a small inclination angle with respect to the TB in grain 5. As shown in Figs. 5 (b) - (h), strain accumulation is observed adjacent and parallel to the TB at early stages of the fatigue loading and the strain band IJ terminates at the grain boundaries (similar to strain band $\mathrm{AB}$ in Fig. 4). It appears that this impingment of strain band at the grain boundary does not cause a strain enhancement adjacent to the grain boundary. A semi-quantitative assessment of the evolution of maxium shear strain $\varepsilon_{x y}^{\max }$ in the strain band IJ is shown in Fig. 5 (j), from which we can see a slight strain increase after 20000 cycles after experiencing a saturation stage, and then a more evident strain increase after 23000 cycles. After the test was stopped at 31000 cycles, a significant strain increase can be discerned. This strain evolution behaviour is different from strain band $A B$ as shown in Fig. 4. This initial strain saturation and then increase in strain band IJ is asociated with the cracking in this (slip) strain band (i.e. formation of crack 1). More detailed analysis is presented in Fig. 6 and will be discussed later.

Another apparent feature in the maximum shear strain evolution maps is strain band $\mathrm{MN}$ in grain 5, which is associated with crack 2. It was found that this strain band developed continuously with loading cycles accompanied by the development of some secondary strain bands once it appeared. These strain bands are all parallel to the calculated slip traces of $\{111\}$ planes based on EBSD measurement, but they are not all associated with the slip 
systems with high SF. It is also interesting to notice that there is no strain developed adjacent and parallel to the $\mathrm{TB}$ in this grain, indicating the influence of the relative orientation between the TB and the loading direction on strain accumulation/localisation, which is also indicated by the relatively low SF value in this grain. The evolution of strain band $\mathrm{MN}$ in grain 5 is closely related to the propagation of crack 2 . As crack 2 propagates in grain 5 and strain band $\mathrm{MN}$ approaches the grain boundary between grain 5 and grain 3, a new strain band appears in grain 3 (Fig. 5 (g)). As the loading cycle proceeds, crack 2 propagates into grain 3 with little deflection, and crack 1 and crack 2 coalesce as shown in Fig. 5 (i). The initiation site of crack 2 is shown in Fig. 5 (k). It is found that the initiation site is nearby a (hard) boride. Propagation of crack 2 is associated with the extrusion of materials due to the out-of-plane shear as a consequence of out-of-plane dislocation slip, which can be clearly seen in Fig. 5 (1). Apart from the two features associated with crack initiation and propagation, we can also see the strain accumulation at grain boundaries as indicated by yellow arrows in Fig. 5 (c). In addition, a stitching artefact as a result of unexpected stage movement during SEM imaging causes a localised failure of the image correlation process as pointed out in Fig. 5 (b), but this stitching artefact appears to have no effects on the localised strain bands observed in other images. 
(a) before loading

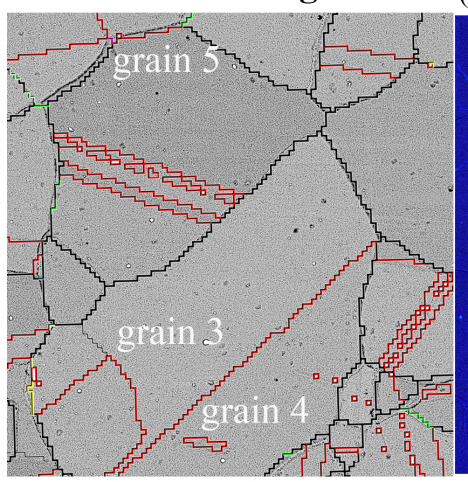

(d) 20000cycles

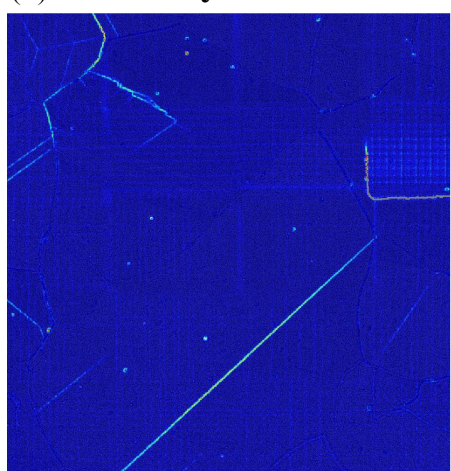

(g) 28000 cycles
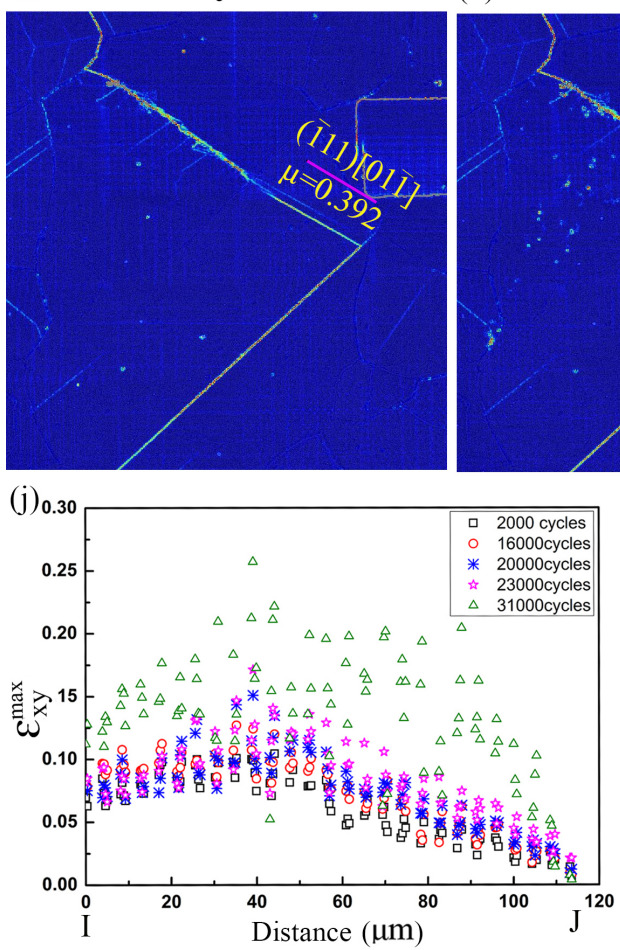

(b) 2000 cycles

(e) 23000cycles

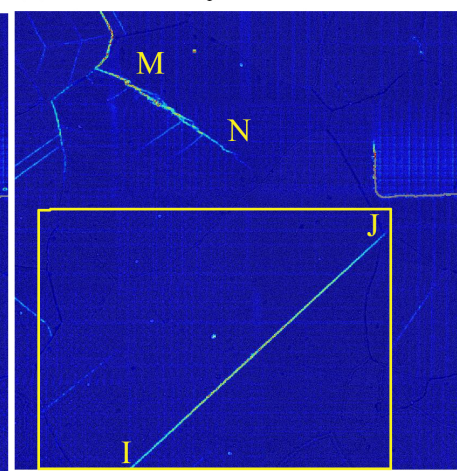

(h) 31000cycles (c) 16000 cycles

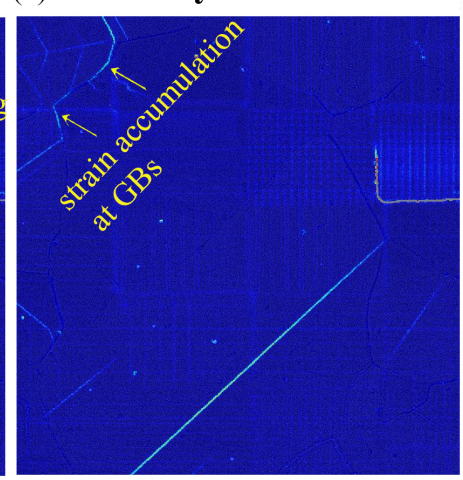

(f) 25000 cycles

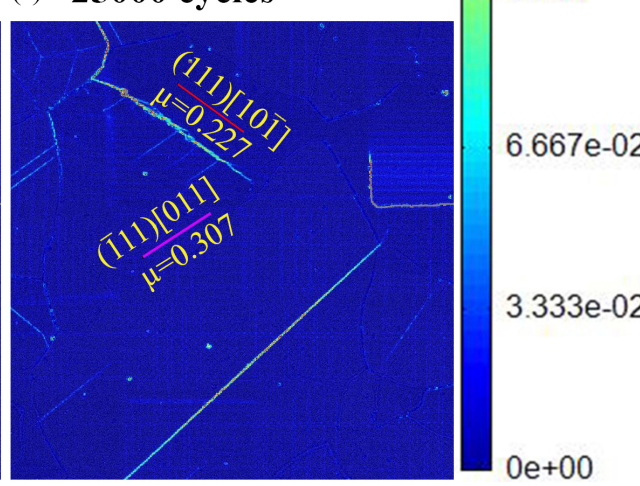

(i) $\mathbf{3 1 0 0 0}$ cycles

$1.333 \mathrm{e}-01$

e-01

$1.667 \mathrm{e}-01$

(1)

$667 e-02$
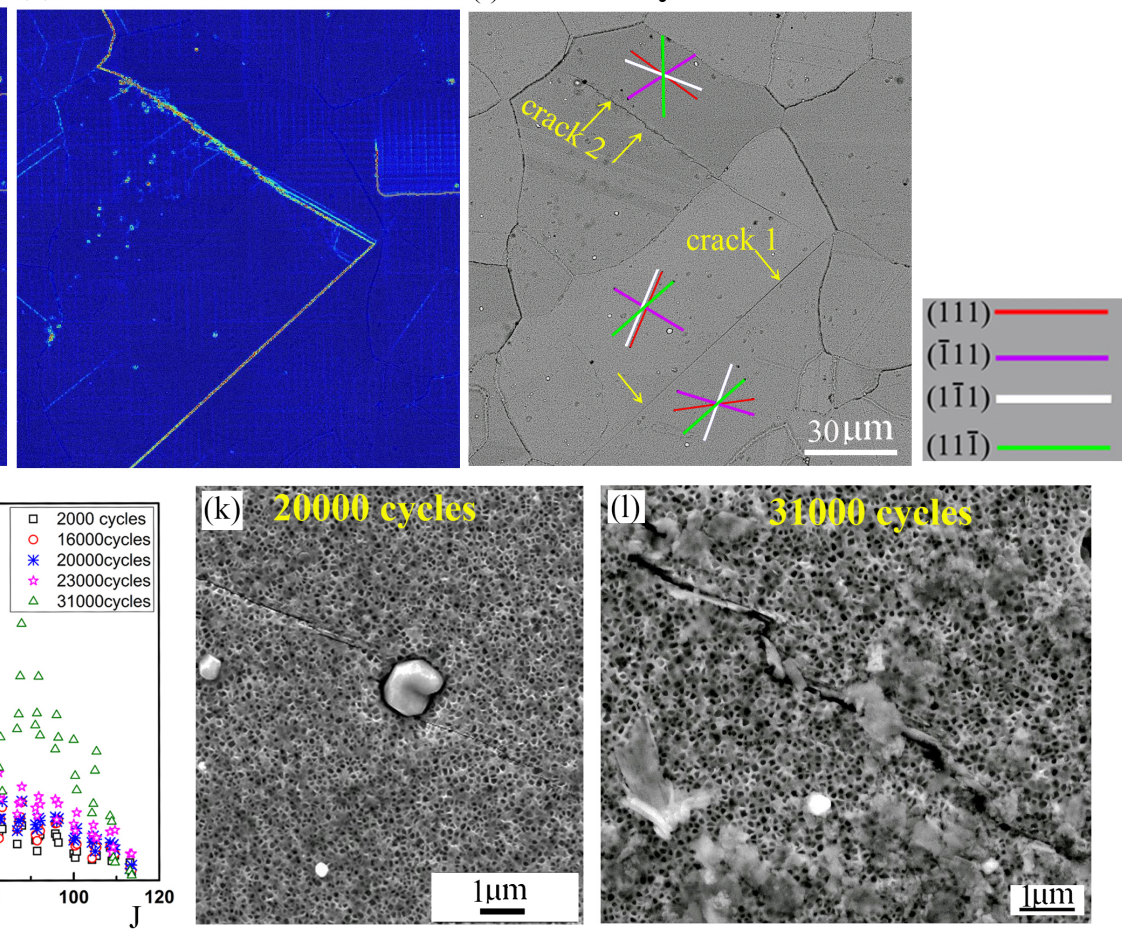

Fig. 5 (a) Stitched BEI image of AOI 7 before fatigue test. High angle grain boundaries (black lines) and twin boundaries (red lines) are overlaid on the BEI image. Maximum shear strain $\varepsilon_{x y}^{\max }$ map at (b) 2000 cycles, (c) 16000 cycles, (d) 20000 cycles, (e) 23000 cycles, (f) 25000 cycles, (g) 28000 cycles and (h) 31000 cycles. (i) Stitched BEI image of AOI 7 at the 
end of the fatigue test. Slip traces of $\{111\}$ planes in grains 3, 4 and 5 are plotted out based on EBSD analysis. (j) Maximum shear strain $\varepsilon_{x y}^{\max }$ distribution in strain band IJ. (k) Crack initiation site of crack 2 observed after 20000 cycles. (1) Material extrusion in the crack path of crack 2 observed at 31000 cycles. For interpretation of the references to colour in this figure legend, the reader is referred to the web version of this article.

Strain distribution in strain band IJ in the region highlighted by the rectangle in Fig. 5 (e) was re-plotted in its local coordinates and is presented in Fig. 6. As shown in Fig. 6 (a), shear strain $\varepsilon_{\mathrm{xy}}$ is dominant in the strain band at 16000 cycles and no evident localised $\varepsilon_{\mathrm{xx}}$ and $\varepsilon_{\mathrm{yy}}$ strain components can be seen in the strain maps. Similar to Fig. 4, this dominant shear strain is associated with the sheared $\gamma^{\prime}$ in the strain band as shown in Fig. 6 (c). Although $\varepsilon_{\mathrm{xy}}$ is still dominant in strain band IJ at 20000 cycles, some $\varepsilon_{\mathrm{yy}}$ can be discerned in a small portion of the strain band as pointed out by red arrows in Fig. 6 (a), which may indicate the occurrence of early cracking in this strain band. A clear cracking in strain band IJ can be observed after 23000 cycles, and an example is shown in Fig. 6 (d), where we can see the coexistence of a crack and sheared $\gamma^{\prime}$. At 31000 cycles, $\varepsilon_{y y}$ can be found in the most of the strain band, and an increased shear strain $\varepsilon_{\mathrm{xy}}$ can also be observed. In addition, some $\varepsilon_{\mathrm{xx}}$ can be discerned. A semi-quantitative strain distribution in strain band IJ in its local coordinates is presented in Fig. 6 (b). For the $\varepsilon_{y y}$ strain component, it can be seen that the value is close to the background noise level before the increase of $\varepsilon_{\mathrm{yy}}$ occurs in a portion of the strain band (which is also shown in Fig. 6 (a)) at 20000 cycles. And then the increase in $\varepsilon_{y y}$ spreads through most of the strain band in the following loading cycles. A significant increase of $\varepsilon_{y y}$ can be seen at 31000 cycles, and this increase is believed to be associated with the crack coalescence which results in a sharp opening of the crack. In terms of $\varepsilon_{\mathrm{xx}}$ and $\varepsilon_{\mathrm{xy}}$, slight but gradual increases can be discerned after cracking occurred in the strain band (i.e. after 20000 cycles), and a significant increase can be seen at 31000 cycles due to coalescence of cracks 1 and 2 shown in Fig. 5. Through this detailed analysis, the onset of cracking in a strain band can be identified and prior to that, it is possible to observe how strain accumulates in a strain band during fatigue cycling whilst relating this to the known grain and twin boundary orientations. The value of such observations/measurements to both initialise and validate crystal plasticity and other microstructural modelling frameworks is clear. 

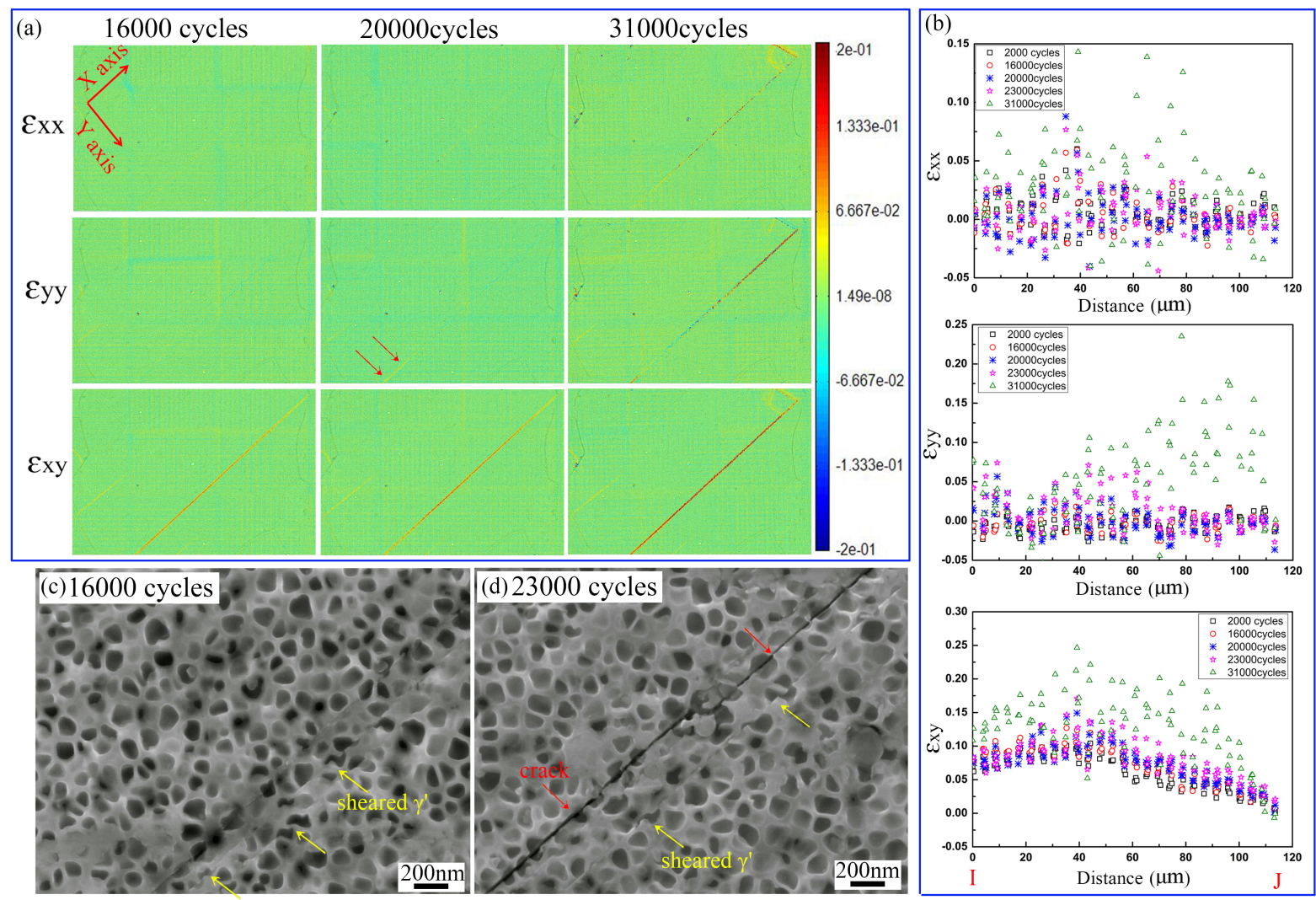

Fig. 6 (a) Strain distribution in strain band IJ in its own local coordinates at 16000, 20000 and 31000 cycles. The $\mathrm{X}$ axis is along the strain band. (b) Evolution of $\varepsilon_{\mathrm{xx}}, \varepsilon_{\mathrm{yy}}$ and $\varepsilon_{\mathrm{xy}}$ with loading cycle in the local coordinates of strain band IJ. (c) Microstructural features in strain band IJ at 16000 cycles, showing sheared $\gamma^{\prime}$. And (d) Microstructural feature in strain band IJ at 23000 cycles, showing the coexistence of sheared $\gamma^{\prime}$ and crack. For interpretation of the references to colour in this figure legend, the reader is referred to the web version of this article.

\subsection{Strain localisation in the early crack propagation regime}

In order to study strain localisation development during the early crack propagation regime, two small notches with dimensions of $30 \mu \mathrm{m} \times 1 \mu \mathrm{m} \times 10 \mu \mathrm{m}$ were prepared by FIB at the top central surface of the plain bend bar specimen so as to have specific targeted areas from where to take the reference images before cracks started to propagate. One of the FIBed notches was fabricated at the TB in a large grain with high SF in AOI-N1, and the other one was fabricated at a TB in a relatively small grain with low SF in AOI-N2 as shown in Fig. 1 (c). The reference and deformed images were taken at 5000× magnification to better detect crack propagation. Fig. 7 presents the maximum shear strain $\varepsilon_{x y}^{\max }$ evolution at the tip of the notch in AOI-N1. Figs. 7 (a) and (b) show the morphology of investigated AOI-N1 before 
and at the end of the fatigue test respectively. Grain boundaries and twin boundaries are overlaid on the stitched BEI image in Fig. 7 (a) along with the calculated slip traces of $\{111\}$ planes according to the EBSD measurements. Due to the relatively large step size (i.e. $2 \mu \mathrm{m}$ ) used for EBSD mapping, some grain boundary segments are less accurately overlaid upon the BEI image. As shown in Fig. 7 (b), a crack initiates from the notch tip adjacent and parallel to the TB and then propagates into neighbouring grains with evident opening displacement in the crack path. There are a few more cracks, one which initiates adjacent and parallel to the $\mathrm{TB}$, and others which mainly initiate from slip bands in grain 8 as indicated by the red arrows.

Figs. 7 (c) - (j) present the maximum shear strain $\varepsilon_{x y}^{\max }$ evolution in the region highlighted by the rectangle shown in Fig. 7 (a), from which it can be seen that the strain evolution is closely related to the crack propagation from the notch tip. Due to the higher magnification used in this case, the whole AOI-N1 shown in Fig. 7 (a) has not been imaged at every interruption/observation of the test. The grain boundaries and twin boundaries are also overlaid on $\varepsilon_{x y}^{\max }$ maps in Figs. 7 (c) and (j) to better visualise the strain (slip) transmission through grain boundaries during crack propagation. It should be noted that the reference images used for this section of DIC analysis were taken after 6000 cycles as the original undeformed images taken before the fatigue test and originally intended as the baseline reference yielded more background noise due to some unexpected SEM stage movement and more significant image drift issues. The DIC analysis carried out between the undeformed material and after 6000 cycles shows limited strain accumulation adjacent and parallel to TB at the notch tip in the investigated AOI-N1 and only a strain localisation adjacent and parallel to the TB in grain 8. It is therefore considered that using reference images from the 6000 cycle point will still yield useful information in terms of the evolving strain distribution throughout the test beyond that point. As shown in Figs. 7 (c) - (j), the strain initially accumulates adjacent and parallel to the TB at the notch tip, and then cracking of the strain band occurs as the loading cycles proceed. As the crack approaches the grain boundary between grains 7 and 8 , a strain band starts to develop in grain 8 . This newly developed strain band at the crack tip in grain 8 is not associated with the slip system with the highest SF based on the calculated slip traces of the $\{111\}$ planes. During further propagation of the crack, we can see the strain accumulation developing at the crack tip. Meanwhile, new strain bands appear and intersect at the crack as shown in Figs. 7 (d) - (f), and the strain in these strain bands increases to some extent as the loading cycle increases (Figs. 7 (g) - (j)). As the crack approaches the boundary between grains 8 and 9, the strain band in the neighbouring 
grain at the crack tip is activated (Fig. 7 (e)). A widening of the strain band in grain 9 at the crack tip and then separation of the strain band can be found as the crack approaches and propagates into grain 9, accompanied by a slight strain accumulation at the grain boundary between grains 8 and 9 (Figs. 7 (g)-(j)). However, it seems that the crack is arrested (or significantly decelerated) by a TB which has a large inclination angle with respect to the crack path. More detailed analysis of the strain accumulation at the crack tip in grain 9 is shown in Fig. 8. It can also be seen that there is a failure in the image correlation along the crack path in Figs. 7 (e) - (j) due to the large crack opening displacement as it approaches and propagates into grain 9. Intensive strain bands are observed adjacent to the crack path in grains 7 and 8 as highlighted by the yellow ellipse in Fig. 7 (j), more detailed analysis of this feature is shown in Fig. 9 and will be discussed later.

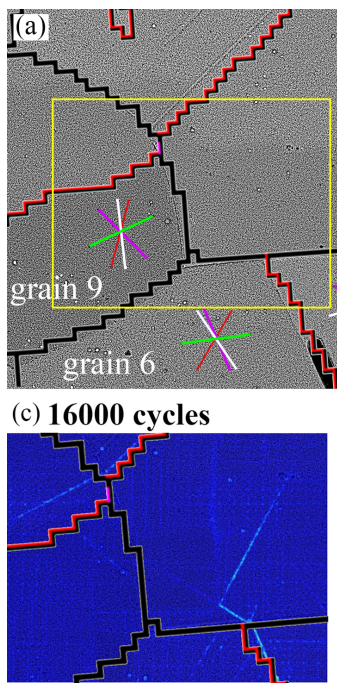

(g) 22000 cycles

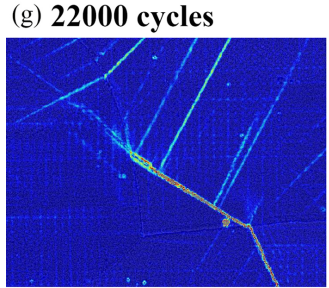

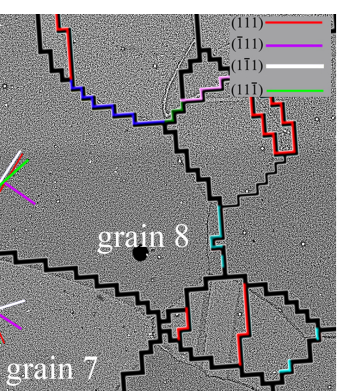

(d) 18000 cycles

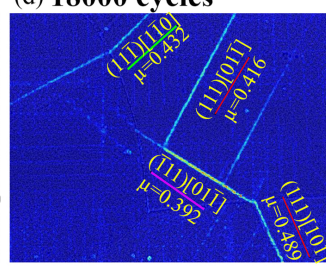

(h) 23000 cycles

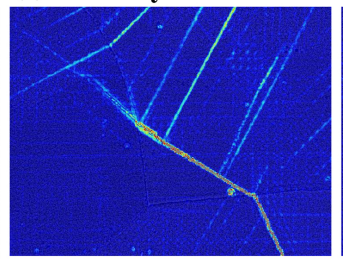

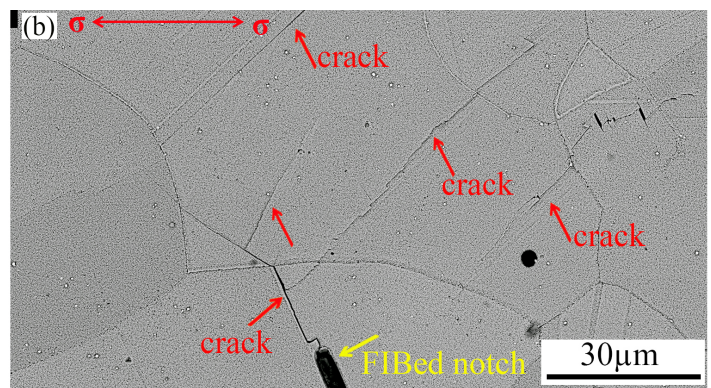

(e) 20000 cycles

(f) 21000 cycles

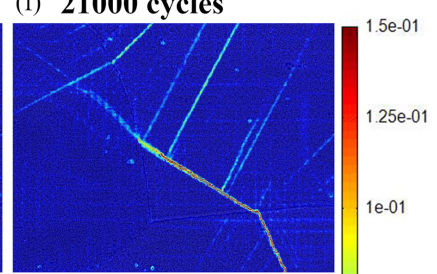

(i) $\mathbf{2 5 0 0 0}$ cycles

(j) 28000 cycles

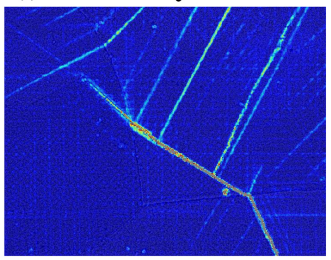

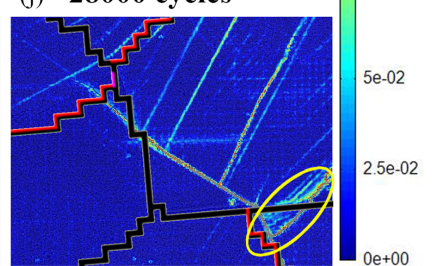

Fig. 7 (a) Stitched BEI image of AOI-N1. High angle GBs (black lines) and TBs (red lines) are overlaid on the BEI image. Slip traces of $\{111\}$ planes in grains 6-9 are plotted out based on EBSD analysis. (b) Stitched BEI image of AOI at the notch tip at the end of the fatigue test. Maximum shear strain $\varepsilon_{x y}^{\max }$ map in the region highlighted by the yellow rectangle in (a) at (c) 16000 cycles, (d) 18000 cycles, (e) 20000 cycles, (e) 23000 cycles, (f) 21000 cycles, (g) 22000 cycles, (h) 23000 cycles, (i) 25000 cycles and (j) 28000 cycles. For interpretation of the references to colour in this figure legend, the reader is referred to the web version of this article. 
Strain distribution in the strain band at the crack tip in grain 9 is presented in its local coordinates and strain components $\varepsilon_{\mathrm{yy}}$ and $\varepsilon_{\mathrm{xy}}$ are shown in Fig. 8 (a). $\varepsilon_{\mathrm{xx}}$ is not presented due to the lack of differences observed in the strain map of $\varepsilon_{\mathrm{xx}}$ with the increasing loading cycles. As shown in Fig. 8 (a), the strain at the crack tip in grain 9 is predominantly shear at 21000 cycles before the crack propagates through the grain boundary. The morphology of the crack tip at 21000 cycles is shown in Fig. 8 (b), from which we can see intensive slip at the crack tip and the slip terminates at the grain boundary. On further loading by another 1000 cycles, we can see the appearance of $\varepsilon_{\mathrm{yy}}$ along with the change in terms of the $\varepsilon_{\mathrm{xy}}$ distribution at the crack tip in grain 9 . The appearance of $\varepsilon_{\mathrm{yy}}$ indicate an opening in the strain band at the crack tip, i.e. we can unambiguously identify that the crack propagates through the grain boundary and reaches into grain 9. Observation of the crack tip under SEM after 22000 cycles confirms the crack propagation into grain 9 as pointed out by the red arrows in Fig. 8 (c). As the loading progresses, $\varepsilon_{\mathrm{yy}}$ spreads out along the strain band at the crack tip in grain 9 until 25000 cycles, where it appears that the crack is temporarily arrested by a TB as shown in the $\varepsilon_{\mathrm{yy}}$ map and the morphology of the crack tip at 28000 cycles (Fig. 8 (d)).

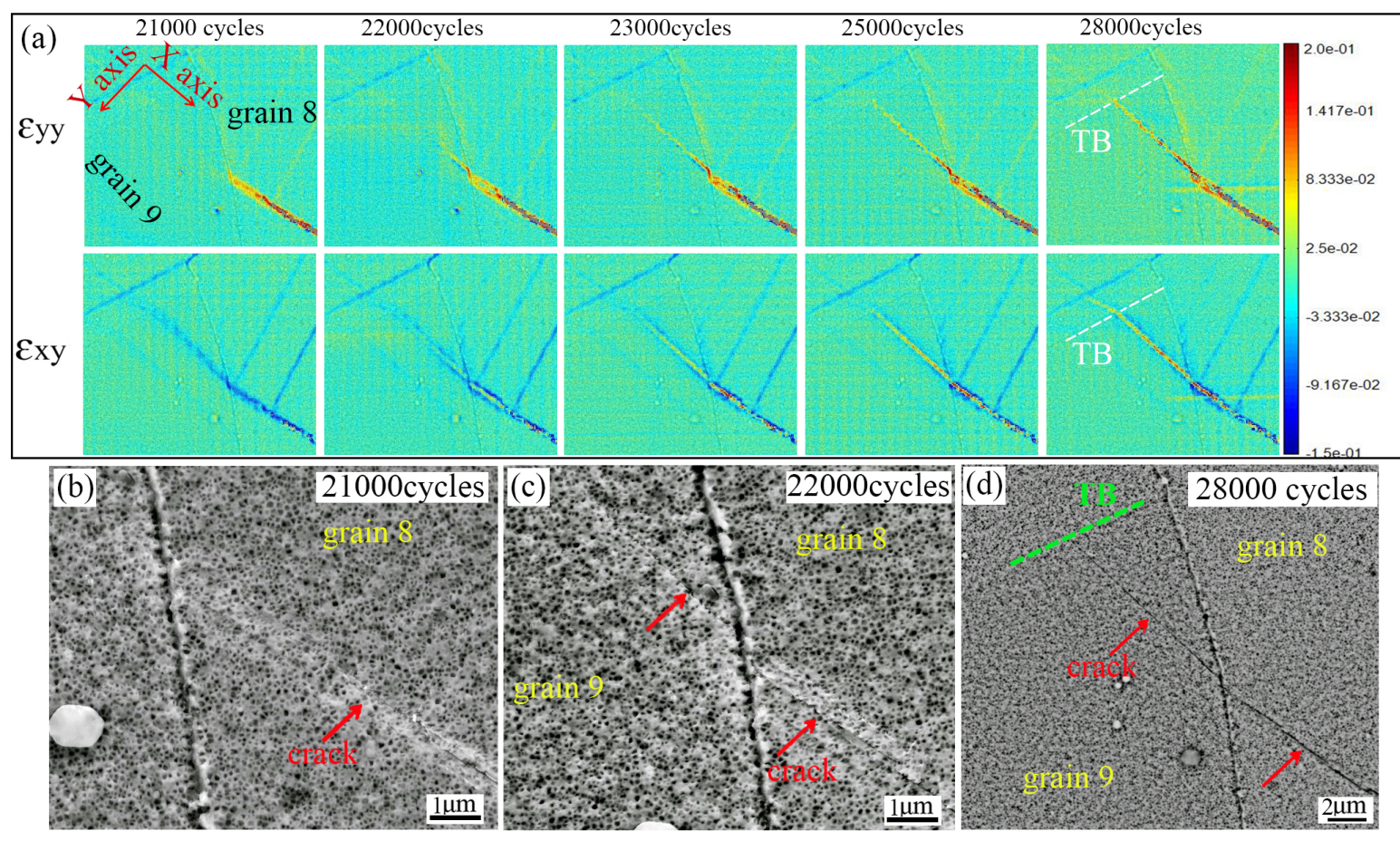

Fig. 8 (a) $\varepsilon_{y y}$ and $\varepsilon_{x y}$ distribution presented in the local coordinates of the strain band at the crack tip in grain 9. Morphology of the crack tip at (b) 21000 cycles, (c) 22000 cycles and (d) 28000 cycles. For interpretation of the references to colour in this figure legend, the reader is referred to the web version of this article. 
Figs. 9 (a) and (b) display the maximum shear strain $\varepsilon_{x y}^{\max }$ distribution in the whole AOI at the tip of notch shown in Fig. 7 (a) at 26000 and 28000 cycles. Intensive strain localisation is discerned in grain 8 which consists of plenty of strain bands apart from the strain bands observed in Fig. 7. These dense strain bands are parallel to the TB and are associated with the slip system with the highest SF as indicated by the calculated slip traces shown in Fig. 7 . The morphology of these intensive strain bands (as highlighted by rectangle 1 in Fig. 9 (b)) at the end of the test is shown in Fig. 9 (c), from which we can see micro-cracking in the strain bands and repeated shear of $\gamma^{\prime}$. At both ends of these intensive strain bands in grain 8 , intensive strain accumulation is also seen in neighbouring grains along with crack propagation into neighbouring grains with a small deflection as shown in Fig. 9 (d). Due to the cracking in these strain bands, it is not possible to correlate the SEM images at these severely deformed regions. It is also interesting to observe that the strain bands and cracks in grains 7 and 10 are close to normal to the loading direction, showing a feature of Stage II crack propagation. Morphology of this seemingly Stage II crack in grain 10 is shown in Fig. 9 (e), which shows micro-cracking along the slip/strain bands nearly normal to the tensile stress direction.
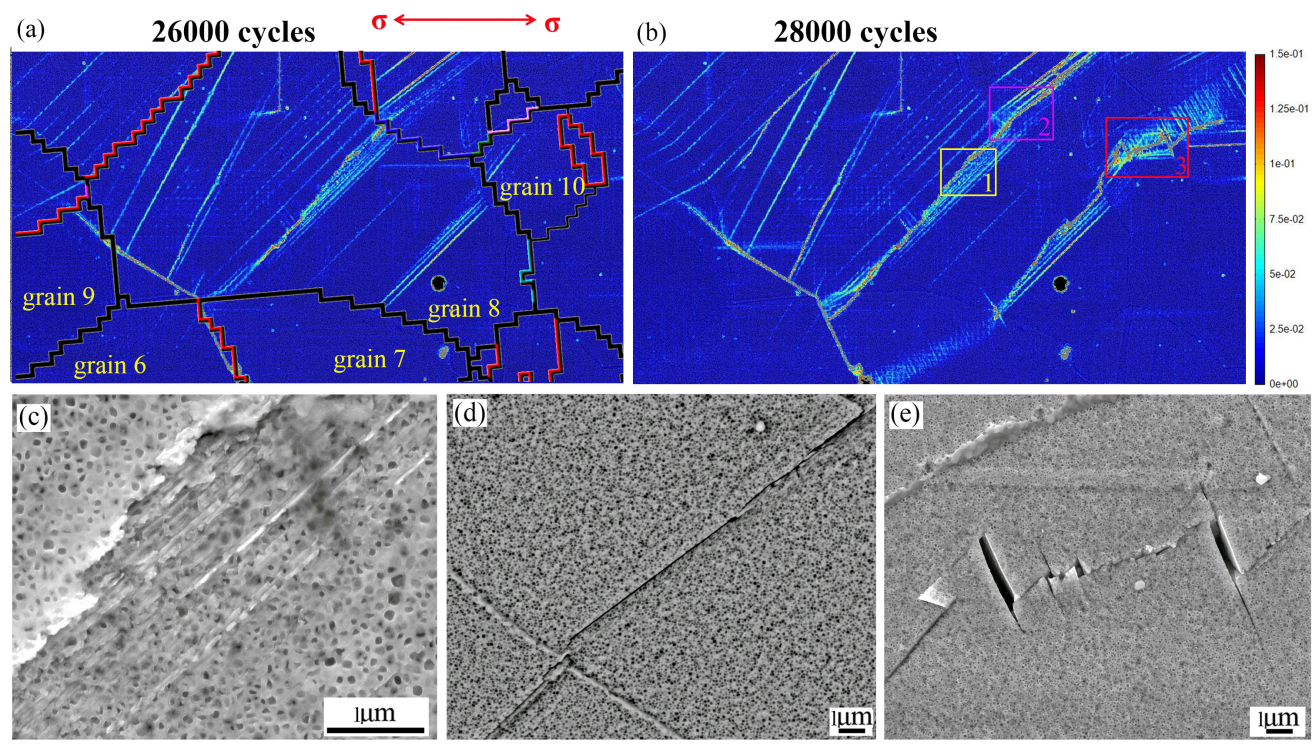

Fig. 9 Maximum shear strain $\varepsilon_{x y}^{\max }$ distribution in the whole AOI-N1 at (a) 26000 and (b) 28000 cycles. High angle grain boundaries (black lines) and twin boundaries (red lines) are overlaid on the $\varepsilon_{x y}^{\max }$ map at 26000 cycles. (c), (d) and (e) show the microstructural features/morphology in the strain bands highlighted in rectangles 1-3 shown in (b). For interpretation of the references to colour in this figure legend, the reader is referred to the web version of this article. 
Fig. 10 shows the strain accumulation at the tip of the notch located at a small grain with relatively low SF in AOI-N2. Unlike the notch in the large grain with high SF, no apparent crack propagation starting from the notch tip is found (Fig. 10 (c)). As shown in maximum shear strain maps in Fig. 10 (a), a few strain bands are observed at the notch tip, but these strain bands are insignificant at 6000 cycles. An evident strain band (i.e. strain band PQ) is located through the whole of grain 13, which is associated with the slip system with the highest SF as indicated by the calculated slip traces. At the end of the test (i.e. 28000 cycles), the strain bands at the notch tip appear more significant and penetrate into the neighbouring grain. The strain band PQ also appears to be more profound. Analysis of strain band PQ in its local coordinates shows that this strain band mainly consists of $\varepsilon_{\mathrm{yy}}$ along with a small amount of $\varepsilon_{\mathrm{xy}}$ as shown in Fig .9 (b). Observation of the notch tip under SEM indicates that the newly appeared strain band in grain 13 is linked to the crack propagation into grain 13 as shown in Fig. 10 (d). But this crack propagation is not significant, and the crack path is associated with the slip system with the lowest SF in this particular grain, indicating here the importance of the crack path geometry (to minimize the crack deflection) on crack propagation. No apparent shear or cracking is found in strain band PQ as shown in Fig. 10 (e).
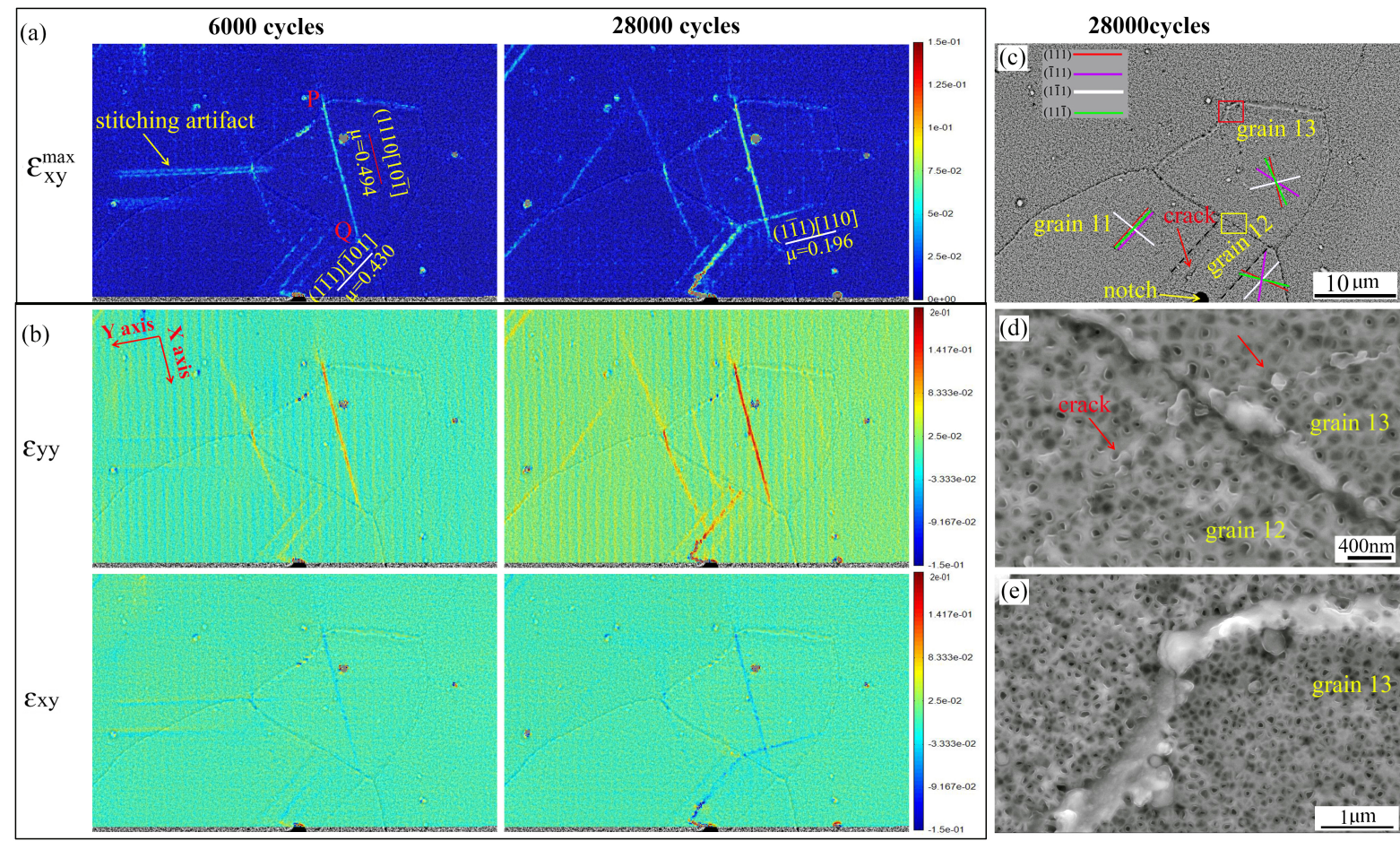

Fig. 10 (a) Maximum shear strain $\varepsilon_{x y}^{\max }$ distribution in AOI-N2 at 6000 and 28000 cycles. (b) $\varepsilon_{\mathrm{yy}}$ and $\varepsilon_{\mathrm{xy}}$ distribution presented in the local coordinates of strain band PQ in grain 13. (c) Morphology of the investigated AOI-N2 at the notch tip at 28000 cycles. The calculated slip 
traces of $\{111\}$ planes are overlaid on the stitched BEI image. The positions of the TB are marked by the black dashed lines. (d) Morphology of crack tip at 28000 cycles in the region highlighted by yellow rectangle in (c). (e) Microstructural features in strain band PQ at 28000 cycles in the region highlighted by red rectangle in (c). For interpretation of the references to colour in this figure legend, the reader is referred to the web version of this article.

\section{Discussion}

\subsection{Strain measurement with ex-situ SEM-DIC}

Strain localisation during the crack initiation regime and at the propagating crack tip in the LSHR alloy has been characterised by SEM-DIC using the secondary $\gamma^{\prime}$ precipitates as speckles. These natural speckles provide a high spatial resolution enabling the mapping of strain distributions with sub-micron strain gauge areas. Due to the ex-situ observation throughout the interrupted fatigue tests, a higher noise level (which is shown as vertical bands in the $\varepsilon_{\mathrm{xx}}$ map and horizontal bands in the $\varepsilon_{\mathrm{yy}}$ map in Fig. 3) was found in this study compared with that in the in-situ tensile tests reported in $[38,52]$. The origin of this kind of noise has not yet been identified. It is reported that the interpolation bias during the image correlation process may produce vertical and horizontal concentrated strain bands $[51,56]$, but it seems that it is not the case in this particular study as this would also appear in DIC maps from images taken during the same session and it does not (see Fig. 2). It is suspected that this noise might be caused by a slight magnification variation during the imaging between different SEM sessions. More work is still required to justify this assumption. In addition, although studies of strain localisation using SEM-DIC in ex-situ tensile tests on steel and Ni-based superalloy [26, 43, 46] and ex-situ compression tests on Ni-based superalloy [38] have been reported, this kind of noise in the form of vertical and horizon bands was not reported. This may be because of the specifics of the particular SEMs used for imaging. However, compared with the localised strain developed under the cyclic load, a good signal to noise ratio is obtained, which makes it possible to perform an effective qualitative analysis on the relative changes in obtained strain fields. Because of the periodic nature of the bands, it would be possible to remove them by using a narrowband filter in Fourier space, though at the risk of removing also some of the signal which lives in the same frequency range. This was therefore not attempted here but may be a solution for future studies.

Although the SEM images obtained for DIC analysis are from ex-situ fatigue tests which introduce more background noise compared with in-situ tests, it seems that the algorithm 
employed can cope with the image translation issues. Also, the ZNSSD algorithm can effectively remove the effects of the differences in contrast between the SEM images obtained at different interruption points throughout the fatigue test. Although the obtained strains are residual in nature, they are large enough to lead to a high signal to noise ratio, which is promising for the application of this ex-situ SEM-DIC technique to other materials. Finally, ex-situ tests provide more flexibility in terms of specimen dimension, load frames, test environments and temperatures compared to in-situ tests, widening the scope of SEMDIC for materials science studies.

\subsection{Slip band or crack?}

Residual strain evolution with loading cycles in the slip bands shows a tendency to saturation before a crack initiates from these slip bands. The strain localisation preferentially occurs adjacent and parallel to the TB in large grains with a high SF in the early stages of the fatigue life. This is consistent with Miao's [8] and Stinville's [9] study on Rene 88DT alloy and our previous study on the LSHR alloy [16]. Due to the elastic anisotropy across the TB, which causes a relatively high localised stress, strain accumulation preferentially occurs around the TB $[8,38,57]$. Depending on the relative orientation between dislocations and TB, dislocations can slip parallel to the TB or transmit through the TB along with a residual dislocation left at TB $[38,39]$. According to the current study as well as in agreement with [38], it seems that a parallel slip configuration is dominant in the strain localisation process. We have now observed that the strain localisation adjacent and parallel to the TB usually results in crack initiation at these regions after a strain saturation stage as the fatigue loading proceeds, as shown in Fig. 5. Once cracking occurs, the strain obtained by DIC tends to increase as the crack propagates, as shown in Figs. 5 and 6, due to the increased crack opening displacement. It is worth noting that over a crack, what is observed is not a 'strain' stricto sensu but a relative crack opening displacement, which does not affect the conclusions made here. However, it should be noted that the DIC correlation coefficient is also related to the crack opening displacement. The DIC algorithm may fail to correlate the deformed to the reference images when the crack opening displacement is too high, as shown in the crack path in Fig. 7.

The obtained full-field strain distribution has been analysed in both global (associated with the loading direction) and local (associated with the slip/strain bands) coordinates. In the local coordinates, the obtained strain can be linked directly to the dislocation slip on the $\{111\}$ planes in Ni-based superalloys. In the crack initiation regime, both shear strain $\varepsilon_{\mathrm{xy}}$ and 
transverse strain $\varepsilon_{\mathrm{yy}}$ (which is normal to the strain band in the local coordinates) are observed depending on the dislocation slip direction on a $\{111\}$ plane. Although DIC is an in-plane strain measurement method, it appears that the out-of-plane deformation can result in transverse strain $\varepsilon_{y y}$ in the local coordinates due to the in-plane stretch as a consequence of slip extrusion associated with out-of-plane dislocation slip. Although TEM characterisation of the dislocation configuration has not been conducted in this study, SEM observations in the strain band usually show sheared $\gamma^{\prime}$ precipitates in the strain band with a feature of nearly pure shear strain, whereas an extrusion is discerned in the strain band with a feature of dominant transverse strain as shown in Fig. 4. Such slip extrusions can be seen more clearly by cross-sectioning the slip band with FIB as shown in the Ni-based superalloy Rene 88DT [38]. To better describe the dislocation slip process and correlate the obtained strain field to the dislocation slip, the inclination angle $\theta$ between the dislocation slip direction on the activated $\{111\}$ plane and the slip trace of the $\{111\}$ plane on the specimen surface (which is in line with the strain band) is defined and can be calculated based on the EBSD measurement. A schematic diagram of the inclination angle $\theta$ is shown in Fig. 11, and $\theta$ can be calculated using Equation (2).

$$
\theta=\left(\bar{n} \times \bar{n}_{s}\right) \cdot \bar{b}
$$

where $\bar{n}$ is the activated slip plane normal, $\bar{n}_{s}$ is the specimen surface normal, and $\bar{b}$ is the Burgers vector in the activated slip system. " $X$ " is the operation of cross product between two vectors. The calculation of the inclination angle $\theta$ was conducted in the crystal coordinates in an individual grain. The activated $\{111\}$ plane can be identified by comparing the observed strain band based on DIC measurement and the calculated slip trace based on EBSD analysis. The Burger vector $\bar{b}$ is chosen from the slip system with the highest SF in the activated $\{111\}$ plane (here only a/2 $<110>$ perfect dislocations are considered for simplicity, although it is well-understood that the deformation mode at low temperature in Ni-based superalloys is usually associated with the super-dislocation which contains two paired perfect dislocations cutting through the $\gamma^{\prime}$ precipitates). The calculated $\theta$ corresponding to the strain bands in grains 1-13 shown in Figs. 4, 5, 7 and 10 as well as other strain bands parallel to the TB in the AOIs shown in Fig. 1 (b) are presented in Table 4 . The mean value of $\varepsilon_{x x}, \varepsilon_{y y}$ and $\varepsilon_{x y}$ in the strain bands are measured once the strain bands appear, and are also shown in Table 4 . The measurement of the mean $\varepsilon_{\mathrm{xx}}, \varepsilon_{\mathrm{yy}}$ and $\varepsilon_{\mathrm{xy}}$ ahead of the crack tip has aimed to avoid the cracking region (with the assistance of SEM observation). The ratio of $\varepsilon_{\mathrm{yy}} / \varepsilon_{\mathrm{xy}}$ in these strain bands is plotted against the $|\cos (\theta)|$ and is shown in Fig. 12. As shown in Table 4 and Fig. 12, 
the strain band with dominant shear strain $\varepsilon_{\mathrm{xy}}$ (i.e. low $\varepsilon_{\mathrm{yy}} / \varepsilon_{\mathrm{xy}}$ ratio) corresponds to inclination angle $\theta$ close to $0^{\circ}$ or $180^{\circ}$ (or $|\cos (\theta)| \sim 1$ ), indicating dominant in-plane shear/dislocation slip. Conversely, the strain band with dominant transverse strain $\varepsilon_{\mathrm{yy}}$ (i.e. high $\varepsilon_{\mathrm{yy}} / \varepsilon_{\mathrm{xy}}$ ratio) without observable cracking corresponds to an inclination angle $\theta$ which is close to $90^{\circ}$ (or $|\cos (\theta)|<0.4$ ), indicating dominant out-of-plane shear/dislocation slip. The observed transverse strain $\varepsilon_{\mathrm{yy}}$ is associated with the in-plane stretch caused by out-of-plane shear/extrusion. This shows that $\varepsilon_{\mathrm{yy}} / \varepsilon_{\mathrm{xy}}$ has a good correlation with the inclination angle $\theta$ $(|\cos (\theta)|)$ calculated by a Schmid factor analysis in general. However, it can be seen that two of the analysed strain bands (as highlighted by red bold font in Table 4 and indicated by red allows in Fig. 12) were plotted against the inclination angle $\theta$ calculated using the slip direction from the slip system with the second highest SF on the activated slip plane. In Table 4 , it can be seen that the highest and second highest SF associated with these two particular strain bands are only slightly different. A Schmid factor analysis considers the slip systems operating in a grain as if it were a single crystal, unaffected by surrounding grains. The agreement seen for these polycrystalline cases is in itself noteworthy and considering the expected constraint from the surrounding grains in polycrystalline materials during deformation, it is expected that the actual resolved stresses may instead activate the slip system with the second highest SF.

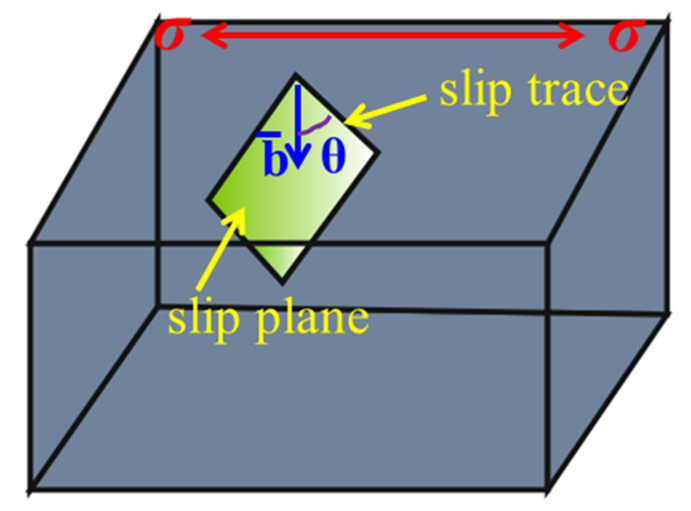

Fig. 11 Schematic diagram of inclination angle $\theta$ between dislocation slip direction on the activated $\{111\}$ plane and the slip trace of $\{111\}$ plane associated with the activated slip system on the specimen surface. 
Table 4 Calculated inclination angle $\theta$ between dislocation slip direction on the activated

$\{111\}$ plane and the slip trace of the $\{111\}$ plane at the specimen surface (corresponding to the strain bands observed by SEM-DIC)

\begin{tabular}{|c|c|c|c|c|c|c|c|}
\hline Grain ID & Slip system & $\begin{array}{l}\text { Schmid } \\
\text { factor }\end{array}$ & $\begin{array}{c}\text { Inclination } \\
\text { angle } \theta\end{array}$ & Exx & Eyy & Exy & $\begin{array}{l}\text { No. of cycles } \\
\text { for the } \\
\text { measurement }\end{array}$ \\
\hline 1 & $(1-11)[-101]$ & 0.491 & 12.6 & 0.0126 & 0.0105 & 0.0482 & 2000 \\
\hline \multirow{2}{*}{2} & $(-111)[101]$ & 0.421 & 103 & \multirow{2}{*}{0.0088} & \multirow{2}{*}{0.0678} & \multirow{2}{*}{0.0117} & \multirow{2}{*}{2000} \\
\hline & $(-111)[01-1]$ & 0.444 & 16.1 & & & & \\
\hline \multirow{2}{*}{3} & $(11-1)[1-10]$ & 0.464 & 13.5 & 0.0072 & 0.0032 & 0.0531 & 2000 \\
\hline & $(-111)[01-1]$ & 0.392 & 32.1 & 0.0140 & 0.0359 & 0.0939 & 28000 \\
\hline \multirow{3}{*}{5} & $(111)[10-1]$ & 0.227 & 118 & 0.0120 & 0.0333 & 0.0282 & 20000 \\
\hline & $(-111)[01-1]$ & 0.307 & 1.32 & \multirow{2}{*}{0.0030} & \multirow{2}{*}{0.0499} & \multirow{2}{*}{0.0157} & \multirow{2}{*}{20000} \\
\hline & $(-111)[110]$ & 0.287 & 58.5 & & & & \\
\hline 7 & $(111)[01-1]$ & 0.489 & 60 & 0.0178 & 0.0343 & 0.0278 & 6000 \\
\hline \multirow{3}{*}{8} & $(111)[01-1]$ & 0.416 & 102 & 0.0190 & 0.0800 & 0.0107 & 16000 \\
\hline & $(-111)[01-1]$ & 0.392 & 42.9 & 0.0178 & 0.0505 & 0.0322 & 16000 \\
\hline & $(11-1)[1-10]$ & 0.432 & 11.7 & 0.0199 & 0.0051 & 0.0680 & 6000 \\
\hline 9 & $(-111)[01-1]$ & 0.474 & 25 & 0.0012 & 0.0038 & 0.0278 & 20000 \\
\hline 12 & $(1-11)[-101]$ & 0.430 & 7.56 & 0.0008 & 0.0144 & 0.0205 & 6000 \\
\hline \multirow{2}{*}{13} & $(111)[10-1]$ & 0.494 & 70.2 & 0.0031 & 0.0736 & 0.0186 & 6000 \\
\hline & $(1-11)[110]$ & 0.196 & 165 & 0.0216 & 0.0050 & 0.0264 & 20000 \\
\hline AOI 1-A1 & $(11-1)[1-10]$ & 0.485 & 16.6 & 0.0077 & 0.0028 & 0.0548 & 2000 \\
\hline AOI 1-A2 & $(11-1)[1-10]$ & 0.469 & 0.41 & 0.0055 & 0.0028 & 0.0198 & 2000 \\
\hline AOI 1-A3 & $(11-1)[1-10]$ & 0.494 & 12.6 & 0.0061 & 0.0044 & 0.0342 & 2000 \\
\hline AOI 2-A4 & $(1-11)[110]$ & 0.496 & 161 & 0.0096 & 0.0067 & 0.0482 & 2000 \\
\hline AOI 2-A5 & $(-111)[01-1]$ & 0.493 & 12.5 & 0.0067 & 0.0125 & 0.0385 & 2000 \\
\hline AOI 2-A6 & $(11-1)[1-10]$ & 0.432 & 23.2 & 0.0113 & 0.0064 & 0.0224 & 2000 \\
\hline AOI 4-A7 & $(11-1)[1-10]$ & 0.478 & 13.4 & 0.0049 & 0.0100 & 0.0497 & 2000 \\
\hline AOI 5-A8 & $(11-1)[1-10]$ & 0.464 & 17.1 & 0.0025 & 0.0028 & 0.0302 & 2000 \\
\hline AOI 6-A9 & $(1-11)[-101]$ & 0.494 & 18.8 & 0.0060 & 0.0084 & 0.0342 & 2000 \\
\hline
\end{tabular}

Note: The red bold font indicates that the inclination angle $\theta$ was calculated using the slip direction from the slip system with the second highest SF. 


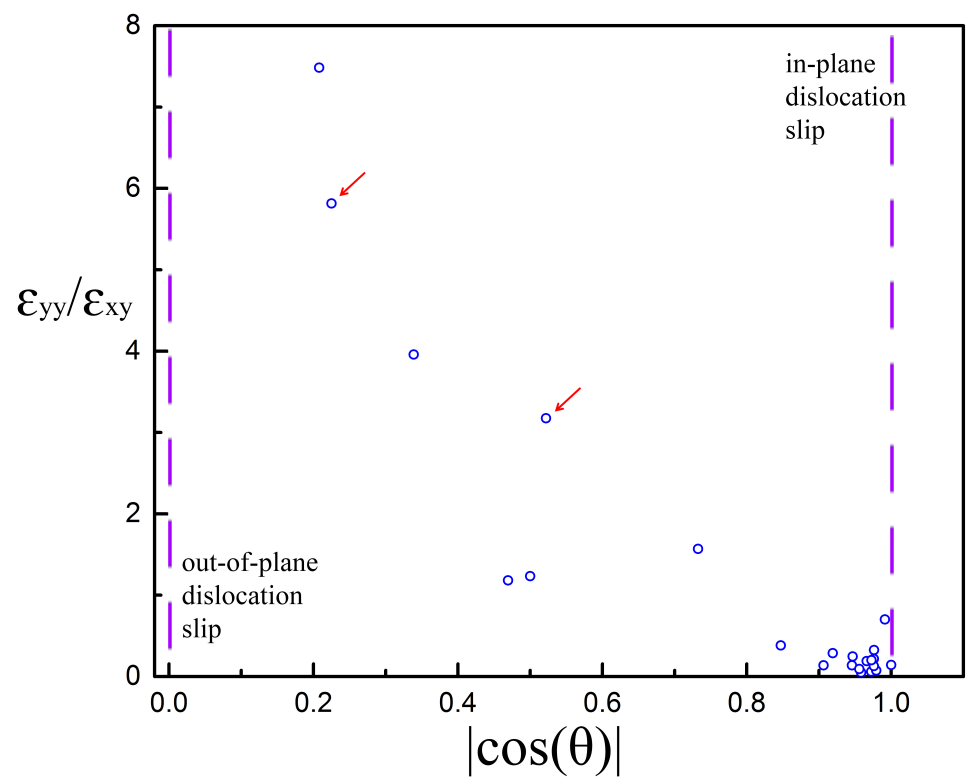

Fig. 12 Relation between the obtained strain measured by SEM-DIC and the predicted type of dislocation slip.

Cracking also causes transverse strain due to the crack opening as shown in Fig. 5. As shown in strain band IJ, the transverse strain starts to appear after a saturation of the shear strain in this strain band associated with mainly in-plane dislocation slip. In this particular strain band, the appearance of transverse strain indicates the occurrence of cracking in the strain band, which is further verified by SEM observation of the morphology in this strain band. It seems that the transverse strain in the full-field strain map can better predict the location of the crack itself than direct SEM and/or OM observation, as it can be detected before the crack opening displacement is large enough for direct microscopy observation as shown in Fig. 6. However, the transverse strain is not exclusively related to cracking, the outof-plane shear/dislocation slip has been seen to also result in transverse strain. By combining the inclination angle $\theta$ between dislocation slip direction and slip trace at surface and the transverse strain in the full-field strain map, it is possible to use these together as a direct cracking indicator. In the case of nearly in-plane shear, the appearance of the transverse strain is usually related to cracking in the strain/slip band, whereas in the case of nearly out-ofplane shear, further direct microscopy observation is necessary to determine the occurrence of cracking.

\subsection{Strain accumulation at the crack tip}

Strain accumulation at the crack tip has been investigated by introducing artificial cracks at TBs in hard-orientated and soft-orientated grains by FIB as the TBs are known as preferential 
sites for fatigue crack initiation at low temperatures. It seems that the FIBed notch can act as a good analogy for the crack, although an initial crack deflection is observed at the notch tip as shown in Figs. 7 and 10. Due to the small size of the FIBed notch, which corresponds to a small plastic zone size, the strain accumulation mainly occurs at the notch tip in the notchcontaining grain. The deformation in the neighbouring grains is not influenced by the introduction of the FIBed notch, i.e. the first observed strain bands in the neighbouring grains are instead associated with the slip systems with the highest SF as shown in Figs. 7 and 10. As the crack approaches a GB, it starts to exert an influence on the deformation in the grains ahead of the crack tip due to the stress concentration effect and the more complex stress state of the plastic zone can encompass the neighbouring grains, which can activate slip systems with an apparently relatively low SF (in terms of the globally applied stress, Figs. 7 and 10). The selection of the crack path ahead of the crack tip depends on both the activated slip systems and the twist/tilt angle of the crack plane when propagating through the grain boundaries. It seems that the twist/tilt angle of the crack plane plays a more important role in crack propagation as it appears that the selection of the crack path tends to minimize this angle based on the observation in this study, which is consistent with Zhai's study [58]. Our previous study on crack path adoption by EBSD also shows that a crack does not propagate exclusively along slip bands with the highest SF slip systems. In some cases, the crack propagates along slip bands with a small inclination angle relative to the previous crack segment, although these slip bands are associated with relatively low SF slip systems in terms of the globally applied stress [16].

Similar to the crack initiation regime, strain accumulation at the crack tip is predominantly shear if the deformation is associated with mainly in-plane dislocation slip as shown in the strain band in grain 9 in Fig. 8 and strain band in grain 13 in Fig. 10. Crack advancement at the crack tip is related to the onset of transverse strain and the observed spread of this transverse strain along the slip/strain band as shown in Fig. 8. Therefore, it may be possible to use the measurement of this transverse strain along with the inclination angle $\theta$ as a clear fracture criterion for crack propagation prediction in CPFE (and other microstructural modelling frameworks). However, the employed DIC parameters are used here to highlight the localised strain, and the obtained strain distribution is so localised that current crystal plasticity models cannot directly predict slip/strain bands as shown in this study at a grain level with sub-micron resolution. In order to bridge the gap between these observed experimental results and current CPFE simulation results, it is possible to increase the subset 
size, step size and the strain window for the DIC analysis to obtain a smoother and more continuous/averaged deformation/strain field (as the CPFE modelling framework assumes). However this also points to the need for different modelling frameworks such as discrete dislocation dynamics approaches to more accurately simulate the physics occurring during crack initiation and propagation in these systems. It should be noted that the current SEMDIC observations, by virtue of the inherent scale of the $\gamma^{\prime}$ speckle, are in fact still providing an averaged (or smoothed) strain value across a larger area compared to the actual dislocation dynamics causing the strain band formation.

\section{Conclusions}

Strain localisation in a P/M Ni-based superalloy, LSHR alloy, has been assessed by SEMDIC using secondary $\gamma^{\prime}$ precipitates as the speckle pattern. Secondary $\gamma^{\prime}$ precipitates in Nibased superalloys provide a fine speckle pattern for DIC analysis to achieve a sub-micron resolution in strain measurement. The obtained strain is semi-qualitative as the absolute values are closely related to the employed DIC parameters, however a useful insight into relative changes in strain levels (and strain components) during the early stages of the fatigue process has been achieved.

SEM-DIC can usefully characterise the detail of strain localisation processes occurring during fatigue crack initiation and early crack propagation regimes. Strain localisation preferentially occurs adjacent and parallel to TBs. In-plane dislocation slip usually results in pure shear strain, and out-of-plane dislocation slip mainly results in transverse strain in the local coordinates associated with the slip band.

Cracking is usually accompanied by an increase in transverse strain $\boldsymbol{\varepsilon}_{\mathbf{y y}}$ in the local coordinates of the slip band. Transverse strain $\boldsymbol{\varepsilon}_{\mathbf{y y}}$ in local slip/strain band coordinates along with the inclination angle $\theta$ (i.e. the inclination angle between the dislocation slip direction on the activated $\{111\}$ plane and the slip trace of the $\{111\}$ plane at the specimen surface) has been shown to be a cracking indicator and can act as a fracture criterion for future CPFE simulations.

Crack propagation is closely related to strain accumulation at the crack tip which is associated with the grain orientation and grain size. Large grains with relatively high SF and long TBs appear to be less resistant to crack propagation compared with small grains with low SF in the short crack propagation regime. Selection of the crack path in an individual 
grain at the crack tip is determined by both the activated slip system and the crack geometry compatibility when the crack propagates into neighbouring grains.

\section{Dataset statement}

The raw data for this paper can be found via the DOI http://doi.org/10.5258/SOTON/D0095

\section{Acknowledgement}

The authors would like to thank Prof. Pascal Lava from KU Leuven for provision of the MatchID software. The authors also would like to thank Dr. Tim Gabb at NASA for supply of LSHR alloy. The EPSRC (Grant no: EP/M000710/1) is gratefully acknowledged for funding support. The authors also would like to thank Dr. Liguo Zhao at the University of Loughborough, Prof. Michael Pruess and Dr. J. Quinta da Fonseca at the University of Manchester, Dr. Mark Hardy at Rolls-Royce, Dr. Gordon McColvin at GE Power and Dr. Matthew Lunt at Defence Science and Technology Laboratory for several useful discussions of the DIC results. Prof. F. Pierron gratefully acknowledges support from the Royal Society and the Wolfson Foundation through a Royal Society Wolfson Research Merit Award. Sari Octaviani gratefully acknowledges support from Schlumberger Faculty for the Future Programme and the Roberto Rocca Education Programme.

\section{Reference}

[1] R.C. Reed, The Superalloys: Fundamentals and Applications, Cambridge University Press, 2006. [2] T.M. Pollock, S. Tin, Nickel-Based Superalloys for Advanced Turbine Engines: Chemistry, Microstructure and Properties , Journal of Propulsion and Power, 22 (2006) 361-374.

[3] R. Jiang, S. Everitt, M. Lewandowski, N. Gao, P.A.S. Reed, Grain size effects in a Ni-based turbine disc alloy in the time and cycle dependent crack growth regimes, International Journal of Fatigue, 62 (2014) 217-227.

[4] H.T. Pang, P.A.S. Reed, Fatigue crack initiation and short crack growth in nickel-base turbine disc alloys - the effects of microstructure and operating parameters, International Journal of Fatigue, 25 (2003) 1089-1099.

[5] H.T. Pang, P.A.S. Reed, Microstructure effects on high temperature fatigue crack initiation and short crack growth in turbine disc nickel-base superalloy Udimet $720 \mathrm{Li}$, Materials Science and Engineering: A, 448 (2007) 67-79.

[6] H.T. Pang, P.A.S. Reed, Effects of microstructure on room temperature fatigue crack initiation and short crack propagation in Udimet $720 \mathrm{Li} \mathrm{Ni-base} \mathrm{superalloy,} \mathrm{International} \mathrm{Journal} \mathrm{of} \mathrm{Fatigue,} 30$ (2008) 2009-2020.

[7] M. Goto, D. M. Knowles, Initiation and propagation behaviour of microcracks in Ni-base superalloy Udimet 720 Li, Engineering Fracture Mechanics, 60 (1998) 1-18.

[8] J. Miao, T.M. Pollock, J. Wayne Jones, Crystallographic fatigue crack initiation in nickel-based superalloy René 88DT at elevated temperature, Acta Materialia, 57 (2009) 5964-5974.

[9] J.C. Stinville, W.C. Lenthe, J. Miao, T.M. Pollock, A combined grain scale elastic-plastic criterion for identification of fatigue crack initiation sites in a twin containing polycrystalline nickelbase superalloy, Acta Materialia, 103 (2016) 461-473. 
[10] Y. Gao, J.S. Stölken, M. Kumar, R.O. Ritchie, High-cycle fatigue of nickel-base superalloy René 104 (ME3): Interaction of microstructurally small cracks with grain boundaries of known character, Acta Materialia, 55 (2007) 3155-3167.

[11] T.P. Gabb, P.T. Kantzos, J. Telesman, J. Gayda, C.K. Sudbrack, B. Palsa, Fatigue resistance of the grain size transition zone in a dual microstructure superalloy disk, International Journal of Fatigue, 33 (2011) 414-426.

[12] R. Jiang, P.A.S. Reed, Critical Assessment 21: oxygen-assisted fatigue crack propagation in turbine disc superalloys, Materials Science and Technology, 32 (2016) 401-406.

[13] R. Jiang, S. Everitt, N. Gao, K. Soady, J.W. Brooks, P.A.S. Reed, Influence of oxidation on fatigue crack initiation and propagation in turbine disc alloy N18, International Journal of Fatigue, 75 (2015) 89-99.

[14] R. Jiang, N. Gao, P.A.S. Reed, Influence of orientation-dependent grain boundary oxidation on fatigue cracking behaviour in an advanced Ni-based superalloy, J Mater Sci, 50 (2015) 4379-4386.

[15] H.S. Kitaguchi, H.Y. Li, H.E. Evans, R.G. Ding, I.P. Jones, G. Baxter, P. Bowen, Oxidation ahead of a crack tip in an advanced Ni-based superalloy, Acta Materialia, 61 (2013) 1968-1981.

[16] R. Jiang, N. Karpasitis, N. Gao, P.A.S. Reed, Effects of microstructures on fatigue crack initiation and short crack propagation at room temperature in an advanced disc superalloy, Materials Science and Engineering: A, 641 (2015) 148-159.

[17] J. Gayda, R.V. Miner, Fatigue crack initiation and propagation in several nickel-base superalloys at $650^{\circ} \mathrm{C}$. International Journal of Fatigue, International Journal of Fatigue, 5 (1983) 135-143.

[18] C.A. Stein, A. Cerrone, T. Ozturk, S. Lee, P. Kenesei, H. Tucker, R. Pokharel, J. Lind, C. Hefferan, R.M. Suter, A.R. Ingraffea, A.D. Rollett, Fatigue crack initiation, slip localization and twin boundaries in a nickel-based superalloy, Current Opinion in Solid State and Materials Science, 18 (2014) 244-252.

[19] W. Schaef, M. Marx, H. Vehoff, A. Heckl, P. Randelzhofer, A 3-D view on the mechanisms of short fatigue cracks interacting with grain boundaries, Acta Materialia, 59 (2011) 1849-1861.

[20] B. Lin, L.G. Zhao, J. Tong, H.J. Christ, Crystal plasticity modeling of cyclic deformation for a polycrystalline nickel-based superalloy at high temperature, Materials Science and Engineering: A, 527 (2010) 3581-3587.

[21] A. Karabela, L.G. Zhao, B. Lin, J. Tong, M.C. Hardy, Oxygen diffusion and crack growth for a nickel-based superalloy under fatigue-oxidation conditions, Materials Science and Engineering: A, 567 (2013) 46-57.

[22] F. Farukh, L.G. Zhao, R. Jiang, P. Reed, D. Proprentner, B.A. Shollock, Realistic microstructurebased modelling of cyclic deformation and crack growth using crystal plasticity, Computational Materials Science, 111 (2016) 395-405.

[23] T. Zhang, D.M. Collins, F.P.E. Dunne, B.A. Shollock, Crystal plasticity and high-resolution electron backscatter diffraction analysis of full-field polycrystal Ni superalloy strains and rotations under thermal loading, Acta Materialia, 80 (2014) 25-38.

[24] S.R. Yeratapally, M.G. Glavicic, M. Hardy, M.D. Sangid, Microstructure based fatigue life prediction framework for polycrystalline nickel-base superalloys with emphasis on the role played by twin boundaries in crack initiation, Acta Materialia, 107 (2016) 152-167.

[25] M.D. Sangid, The physics of fatigue crack initiation, International Journal of Fatigue, 57 (2013) $58-72$.

[26] F. Di Gioacchino, J. Quinta da Fonseca, An experimental study of the polycrystalline plasticity of austenitic stainless steel, International Journal of Plasticity, 74 (2015) 92-109.

[27] N. Jia, Z.H. Cong, X. Sun, S. Cheng, Z.H. Nie, Y. Ren, P.K. Liaw, Y.D. Wang, An in situ highenergy X-ray diffraction study of micromechanical behavior of multiple phases in advanced highstrength steels, Acta Materialia, 57 (2009) 3965-3977.

[28] S. Harjo, Y. Tomota, M. Ono, Measurements of thermal residual elastic strains in ferriteaustenite $\mathrm{Fe}-\mathrm{Cr}-\mathrm{Ni}$ alloys by neutron and X-ray diffractions, Acta Materialia, 47 (1998) 353-362.

[29] P. Rangaswamy, M.B. Prime, M. Daymond, M.A.M. Bourke, B. Clausen, H. Choo, N. Jayaraman, Comparison of residual strains measured by X-ray and neutron diffraction in a titanium (Ti-6Al-4V) matrix composite, Materials Science and Engineering: A, 259 (1999) 209-219. 
[30] E.W. Huang, R.I. Barabash, Y. Wang, B. Clausen, L. Li, P.K. Liaw, G.E. Ice, Y. Ren, H. Choo, L.M. Pike, D.L. Klarstrom, Plastic behavior of a nickel-based alloy under monotonic-tension and lowcycle-fatigue loading, International Journal of Plasticity, 24 (2008) 1440-1456.

[31] M. Kamaya, A.J. Wilkinson, J.M. Titchmarsh, Quantification of plastic strain of stainless steel and nickel alloy by electron backscatter diffraction, Acta Materialia, 54 (2006) 539-548.

[32] A.J. Wilkinson, G. Meaden, D.J. Dingley, High-resolution elastic strain measurement from electron backscatter diffraction patterns: New levels of sensitivity, Ultramicroscopy, 106 (2006) 307313.

[33] P.S. Karamched, A.J. Wilkinson, High resolution electron back-scatter diffraction analysis of thermally and mechanically induced strains near carbide inclusions in a superalloy, Acta Materialia, 59 (2011) 263-272.

[34] T.B. Britton, J. Jiang, Y. Guo, A. Vilalta-Clemente, D. Wallis, L.N. Hansen, A. Winkelmann, A.J. Wilkinson, Tutorial: Crystal orientations and EBSD - Or which way is up?, Materials Characterization, 117 (2016) 113-126.

[35] T. Zhang, J. Jiang, B.A. Shollock, T.B. Britton, F.P.E. Dunne, Slip localization and fatigue crack nucleation near a non-metallic inclusion in polycrystalline nickel-based superalloy, Materials Science and Engineering: A, 641 (2015) 328-339.

[36] J. Jiang, J. Yang, T. Zhang, F.P.E. Dunne, T.B. Britton, On the mechanistic basis of fatigue crack nucleation in Ni superalloy containing inclusions using high resolution electron backscatter diffraction, Acta Materialia, 97 (2015) 367-379.

[37] A.J. Wilkinson, T.B. Britton, Strains, planes, and EBSD in materials science, Materials Today, 15 (2012) 366-376.

[38] J.C. Stinville, N. Vanderesse, F. Bridier, P. Bocher, T.M. Pollock, High resolution mapping of strain localization near twin boundaries in a nickel-based superalloy, Acta Materialia, 98 (2015) 29-42. [39] W.Z. Abuzaid, M.D. Sangid, J.D. Carroll, H. Sehitoglu, J. Lambros, Slip transfer and plastic strain accumulation across grain boundaries in Hastelloy X, Journal of the Mechanics and Physics of Solids, 60 (2012) 1201-1220.

[40] A. Tatschl, O. Kolednik, A new tool for the experimental characterization of micro-plasticity, Materials Science and Engineering: A, 339 (2003) 265-280.

[41] P. Bing, Q. Kemao, X. Huimin, A. Anand, Two-dimensional digital image correlation for inplane displacement and strain measurement: a review, Measurement Science and Technology, 20 (2009) 062001.

[42] J.D. Carroll, W. Abuzaid, J. Lambros, H. Sehitoglu, High resolution digital image correlation measurements of strain accumulation in fatigue crack growth, International Journal of Fatigue, 57 (2013) 140-150.

[43] F. Di Gioacchino, J. Quinta da Fonseca, Plastic Strain Mapping with Sub-micron Resolution Using Digital Image Correlation, Exp Mech, 53 (2013) 743-754.

[44] J.L. Walley, R. Wheeler, M.D. Uchic, M.J. Mills, In-Situ Mechanical Testing for Characterizing Strain Localization During Deformation at Elevated Temperatures, Exp Mech, 52 (2012) 405-416.

[45] W. Abuzaid, H. Sehitoglu, J. Lambros, Plastic strain localization and fatigue micro-crack formation in Hastelloy X, Materials Science and Engineering: A, 561 (2013) 507-519.

[46] G. Pataky, H. Sehitoglu, Experimental Methodology for Studying Strain Heterogeneity with Microstructural Data from High Temperature Deformation, Exp Mech, 55 (2015) 53-63.

[47] W.A. Scrivens, Y. Luo, M.A. Sutton, S.A. Collette, M.L. Myrick, P. Miney, P.E. Colavita, A.P. Reynolds, X. Li, Development of Patterns for Digital Image Correlation Measurements at Reduced Length Scales, Exp Mech, 47 (2007) 63-77.

[48] M.A. Sutton, N. Li, D. Garcia, N. Cornille, J.J. Orteu, S.R. McNeill, H.W. Schreier, X. Li, A.P. Reynolds, Scanning Electron Microscopy for Quantitative Small and Large Deformation Measurements Part II: Experimental Validation for Magnifications from 200 to 10,000, Exp Mech, 47 (2007) 789-804.

[49] M.A. Sutton, N. Li, D.C. Joy, A.P. Reynolds, X. Li, Scanning Electron Microscopy for Quantitative Small and Large Deformation Measurements Part I: SEM Imaging at Magnifications from 200 to 10,000, Exp Mech, 47 (2007) 775-787. 
[50] M.A. Tschopp, B.B. Bartha, W.J. Porter, P.T. Murray, S.B. Fairchild, Microstructure-Dependent Local Strain Behavior in Polycrystals through In-Situ Scanning Electron Microscope Tensile Experiments, Metall and Mat Trans A, 40 (2009) 2363-2368.

[51] A.D. Kammers, S. Daly, Digital Image Correlation under Scanning Electron Microscopy: Methodology and Validation, Exp Mech, 53 (2013) 1743-1761.

[52] J.C. Stinville, M.P. Echlin, D. Texier, F. Bridier, P. Bocher, T.M. Pollock, Sub-Grain Scale Digital Image Correlation by Electron Microscopy for Polycrystalline Materials during Elastic and Plastic Deformation, Exp Mech, 56 (2015) 197-216.

[53] S. Suresh, Fatigue of Materials, Cambridge University Press, Cambridge, 1998.

[54] H.W. Schreier, M.A. Sutton, Systematic errors in digital image correlation due to undermatched subset shape functions, Exp Mech, 42 (2002) 303-310.

[55] P. Lava, F. Pierron, P.L. Reu, http://diccourse.matchid.org/, (2016).

[56] H.W. Schreier, J.R. Braasch, M.A. Sutton, Systematic errors in digital image correlation caused by intensity interpolation, OPTICE, 39 (2000) 2915-2921.

[57] A. Heinz, P. Neumann, Crack initiation during high cycle fatigue of an austenitic steel, Acta Metallurgica et Materialia, 38 (1990) 1933-1940.

[58] T. Zhai, A.J. Wilkinson, J.W. Martin, A crystallographic mechanism for fatigue crack propagation through grain boundaries, Acta Materialia, 48 (2000) 4917-4927. 